\title{
Electrical Conductivity of the Neutron Star Crust at Low Temperatures
}

\author{
A. I. Chugunov* \\ Ioffe Physical-Technical Institute of the Russian Academy of Sciences, \\ Politekhnicheskaya ul. 26, St. Petersburg, 194021 Russia
}

Received April 20, 2011

\begin{abstract}
The electrical conductivity of the neutron star crust at low temperatures is calculated by taking into account the mixing of the electron wave functions due to the interaction with the crystal lattice of atomic nuclei. We show that the previously existed model of exponential reduction of the electron-ion scattering rate can lead to an overestimation of the electrical conductivity by several orders of magnitude. We propose a simple interpolation formula for use in applications that joins the previously known results of calculating the electrical conductivity at high temperatures with the low-temperature asymptotics found here.
\end{abstract}

DOI: $10.1134 / \mathrm{S} 1063773712010021$

Keywords: neutron stars, transport coefficients.

\section{INTRODUCTION}

Knowing the electrical conductivity of neutron star crusts is important in modeling many of the processes in neutron stars, such as the ohmic decay of the magnetic field and the related crustal heating (see, e.g., Pons et al. 2009; Popov et al. 2010), the current propagation in the neutron star crust through the currentinduced pulsar spin-down mechanism (see, e.g., Beskin and Nokhrina 2007), and the decay of magnetic field inhomogeneities important for the gamma-ray emission from pulsars (Barsukov et al. 2006, 2007, 2009).

Electrons are the main charge carriers in a neutron star crust, while ions are their main scatterers (see, e.g., Potekhin et al. 1999). At low temperatures considered here, ions form a crystal lattice and it is convenient to describe the electron-ion scattering in terms of phonon emission and absorption. These processes are also called electron-phonon scattering. In this paper, for simplicity, the electrical conductivity is calculated by assuming the absence of a magnetic field and is considered to be isotropic.

Up to now, reliable calculations of the electron transport coefficients have been performed for fairly high temperatures (Flowers and Itoh 1976; Baiko and Yakovlev 1995, 1996; Baiko et al. 1998; Potekhin et al. 1999; Chugunov and Yakovlev 2005), when the electron-phonon scattering is efficient on the entire Fermi surface and the electrons may be considered to

*E-mail: andr.astro@mail.ioffe.ru be almost free. In this case, the so-called electronphonon Umklapp processes make a major contribution to the scattering. In these processes, the reciprocal lattice vector is present in the quasi-momentum conservation law (see, e.g., Ziman 1960), which leads to a significant change in the direction of the electron velocity during scattering. At low temperatures $T$, only the short-wavelength acoustic photons with fairly low frequencies $\omega \sim k_{\mathrm{B}} T / \hbar$, where $\hbar$ is the Planck constant and $k_{\mathrm{B}}$ is the Boltzmann constant, have nonexponentially small occupation numbers. Therefore, the electron quasi-momentum changes in Umklapp processes almost exactly by the reciprocal lattice vector g. Together with the energy conservation law, this restricts the scattering region to the neighborhoods of the boundaries of the Brillouin zones, where the electron states are significantly distorted by the interaction with the crystal lattice and gaps appear in the dispersion relation for electrons at the boundaries themselves (see, e.g., Raikh and Yakovlev 1982; Ziman 1960). Such a distortion leads to reduction of the electron scattering in Umklapp processes that has been previously taken into account only approximately - an exponential reduction of the scattering rate at low temperature leading to an exponential growth of the electrical conductivity was introduced on the basis of a simplified model (see, e.g., Gnedin et al. 2001). Such freezing actually takes place in many terrestrial conductors in which the Fermi surface does not intersect with the boundaries of the Brillouin zones and, hence, there exists a minimum wave vector of the phonon that can be involved in the Umklapp process (see, e.g., Fig. 117 
in the book by Ziman (1960)). In contrast, the Fermi surface in a neutron star crust intersects with the boundaries of the Brillouin zones. This is due to the large number of free electrons per atomic nucleus. Accordingly, phonons with an arbitrarily low momentum can be involved in the electron scattering (see Section 4), which, in turn, leads to a power-law temperature dependence of the electrical conductivity. In this paper, we calculate the electrical conductivity by properly taking into account the distortion of the electron states by the crystal lattice.

The paper is structured as follows. In Section 2, we describe the composition of the neutron star crust and introduce basic parameters. In Section 3, we describe the electron states near the boundaries of the Brillouin zones that are used in Section 4 to derive an expression for the electrical conductivity in the neutron star crust at low temperatures. In Section 5, we show that the derived expression in the hightemperature limit reproduces the well-known electrical conductivity calculated in the approximation of free electrons (see, e.g., Flowers and Itoh 1976; Raikh and Yakovlev 1982; Baiko and Yakovlev 1995). The low-temperature asymptotics of the electrical conductivity is derived in Section 6. In Section 7, we propose the interpolation formula (39) that smoothly joins the low-temperature asymptotics derived here with the most accurate calculations of the electrical conductivity at higher temperatures (Potekhin et al. 1999; Gnedin et al. 2001), when the influence of the gaps may be neglected. In the same section, the electrical conductivity of a neutron star crust with a smooth equilibrium nuclear composition (see the Appendix in the monograph by Haensel et al. (2007)) is used an example to illustrate the results. In the final section 8 , we formulate our conclusions and describe the plans for further studies.

\section{PARAMETERS OF THE MATTER}

The neutron star crust is separated into the outer one composed of atomic nuclei (ions) and degenerate electrons and the inner one $\left(\rho \gtrsim 4 \times 10^{11} \mathrm{~g} \mathrm{~cm}^{-3}\right)$ where degenerate neutrons not localized in atomic nuclei are additionally present (see, e.g., Haensel et al. 2007). For simplicity, we will assume that all atomic nuclei at a given density $\rho$ are identical. We will denote the number of nucleons bound in an atomic nucleus by $A_{\text {nuc. }}$. In the inner crust, this number should be distinguished from $A$-the total number of nucleons per nucleus. At densities $\rho \lesssim$ $10^{14} \mathrm{~g} \mathrm{~cm}^{-3}$, all protons are bound in atomic nuclei and determine the nuclear charge $Z e$. Since the binding energy of the nucleons in the neutron star crust is low compared to their rest energy, the number density of atomic nuclei can be estimated as $n_{\mathrm{i}}=\rho /\left(A m_{\mathrm{u}}\right)$, where $m_{\mathrm{u}}=1.6605 \times 10^{-24} \mathrm{~g}$ is the atomic mass unit. The quasi-neutrality condition specifies the electron number density $n_{\mathrm{e}}=n_{\mathrm{i}} Z$.

It is convenient to describe the state of degenerate electrons by the Fermi momentum $p_{\mathrm{F}}$ or Fermi wave number $k_{\mathrm{F}}$ :

$$
p_{\mathrm{F}} \equiv \hbar k_{\mathrm{F}}=\hbar\left(3 \pi^{2} n_{\mathrm{e}}\right)^{1 / 3}=m_{\mathrm{e}} c x_{\mathrm{r}},
$$

where $m_{\mathrm{e}}$ is the electron mass, $x_{\mathrm{r}} \approx 100.9\left(\rho_{12} Z / A\right)^{1 / 3}$ is the electron relativity parameter, and $\rho_{12}$ is the density in units of $10^{12} \mathrm{~g} \mathrm{~cm}^{-3}$. In this paper, we will assume that the electrons are ultrarelativistic $\left(x_{\mathrm{r}} \gg 1\right)$. This approximation is valid at the densities of interest to us, $\rho \gtrsim 10^{10} \mathrm{~g} \mathrm{~cm}^{-3}$. The degeneracy temperature for such electrons is

$$
T_{\mathrm{F}}=\left(\epsilon_{\mathrm{F}}-m_{\mathrm{e}} c^{2}\right) / k_{\mathrm{B}} \approx 5.93 \times 10^{9} x_{\mathrm{r}} \mathrm{K},
$$

where we introduced the electron Fermi energy

$$
\epsilon_{\mathrm{F}} \equiv m_{\mathrm{e}} c^{2} \sqrt{1+x_{\mathrm{r}}^{2}} \approx m_{\mathrm{e}} x_{\mathrm{r}} c^{2} .
$$

In this paper, we investigate matter at $T \ll T_{\mathrm{F}}$.

The crystallization of ions with the formation of a body-centered cubic (bcc) lattice is commonly assumed to be energetically most favorable in the neutron star crust. However, a face-centered cubic (fcc) lattice has almost the same energy (see, e.g., Baiko 2002). Below, we will consider the lattices of both types and will show that the electrical conductivity at low temperatures is weakly sensitive to the type of crystal lattice. Note that a similar conclusion for the case of high temperatures was reached by Potekhin et al. (1999). We will consider matter at temperatures $T \lesssim 10^{8} \mathrm{~K}$ that are lower than the melting temperature, which allows the dynamics of ions to be described in the language of phonons in a crystal. It is convenient to measure the phonon wave numbers in radii of a sphere (in $k$ space) equivalent to the first Brillouin zone $q_{\mathrm{BZ}}=\left(6 \pi^{2} n_{\mathrm{i}}\right)^{1 / 3}$. Note that in the neutron star crust, there are many free electrons per nucleus $(Z \gg 1)$ and $q_{\mathrm{BZ}}$ is small compared to the diameter of the Fermi sphere $2 k_{\mathrm{F}}: 2 k_{\mathrm{F}} / q_{\mathrm{BZ}}=$ $(4 Z)^{1 / 3} \gg 1$. For example, at typical charges of atomic nuclei in the neutron star crust $Z \sim 30$, the ratio $2 k_{\mathrm{F}} / q_{\mathrm{BZ}} \sim 5$.

The quantum effects in the system of ions become important at $T \lesssim T_{\mathrm{p}} / 3$, where

$$
T_{\mathrm{p}}=\frac{\hbar \omega_{\mathrm{p}}}{k_{\mathrm{B}}} \approx 7.832 \times 10^{9}\left(\frac{Z^{2}}{A A_{\text {nuc }}}\right)^{1 / 2} \rho_{12}^{1 / 2} \mathrm{~K}
$$

is the ion plasma temperature, $\omega_{\mathrm{p}}=\left(4 \pi Z^{2} e^{2} n_{\mathrm{i}} / m_{\mathrm{i}}\right)^{1 / 2}$ is the ion plasma frequency. The density dependence of $T_{\mathrm{p}}$ (dotted line) for the inner neutron star crust with a smooth nuclear composition (see Appendix B in 
the book by Haensel et al. (2007)) is presented in Fig. 1. The melting temperature $T_{\mathrm{m}}$ exceeds $T_{\mathrm{p}}$ for the parameters shown in the figure. The quantities $E_{\text {gap }} / 2, T_{\mathrm{U}}$, and $\tilde{T}_{\mathrm{U}}$ are described in Section 3.

\section{ELECTRONS NEAR THE BOUNDARIES OF THE BRILLOUIN ZONES}

Since the temperatures $T \lesssim 10^{8} \mathrm{~K}$ considered here are more than an order of magnitude lower than $T_{\mathrm{p}}$, only low-frequency phonons $\left(\omega \sim k_{\mathrm{B}} T / \hbar \ll \omega_{\mathrm{p}}\right)$ are excited in the crystal. These phonons have small wave numbers and efficiently interact only with the electrons whose states can be significantly distorted by the interaction with the static crystal lattice. All of the preceding papers (see, e.g., Potekhin et al. 1999) devoted to the transport in the dense matter of neutron star crusts were restricted to the approximation of free electrons. In this paper, we go beyond this approximation for the first time (as applied to the transport coefficients in dense matter). Therefore, before considering the electrical conductivity, the electron states should be described in detail, which is done in this section. For brevity, $\hbar=1$ here.

We will describe the electron states following Pethick and Thorsson (1997) and use the extended and periodic zone schemes. The periodic zone scheme is convenient when the scattering is considered, because we can eliminate the reciprocal lattice vector $\mathbf{g}$ in the quasi-momentum conservation law and consider the scattering of electrons with close quasi-momenta. On the other hand, the electrons in the neutron star crust are almost free. Therefore, the Fermi surface in the extended zone scheme is nearly spherical (we will call the corresponding sphere of radius $p_{\mathrm{F}}$ in momentum space the Fermi sphere), which is convenient, for example, in the calculations of transport coefficients at high temperatures. In contrast to most of the problems in solid-state physics under terrestrial conditions, using the reduced zone scheme seems unjustified. The point is that the large number of free electrons per atomic nucleus gives rise to a large number of conduction bands (partially filled bands) in each of which the Fermi surface has a complex shape. In this case, each empty band makes its nontrivial contribution to the conductivity, while their number depends significantly on the matter composition. Therefore, in the reduced zone scheme, an independent calculation of the electrical conductivity is actually required for each nuclear charge $Z$, which extremely complicates its investigation. At the same time, in the extended zone scheme, the shape of the Fermi surface does not depend qualitatively on the nuclear charge (a weakly distorted sphere), which allows a unified description at all $Z$.

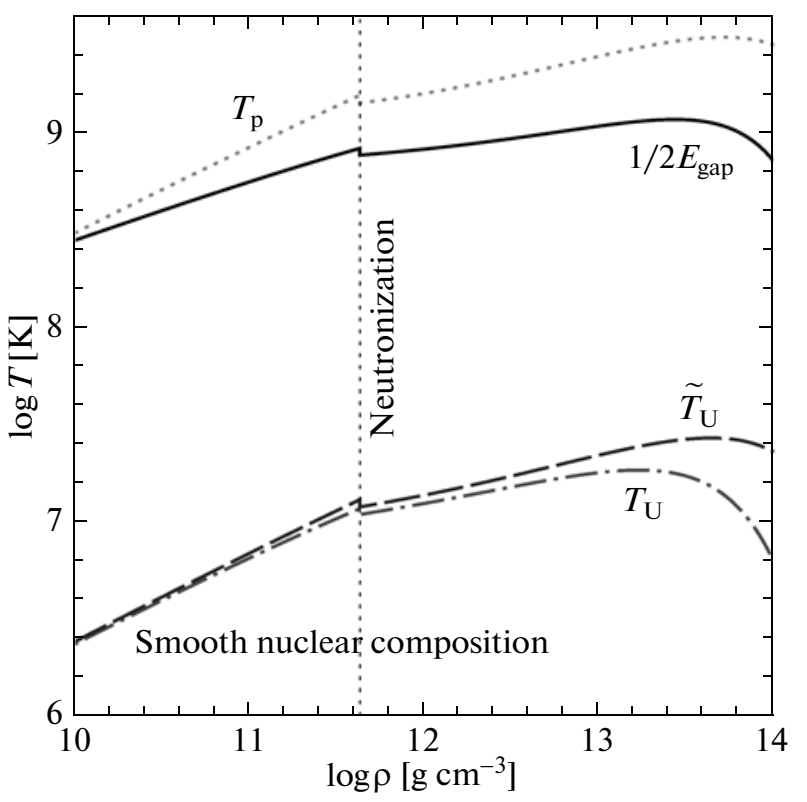

Fig. 1. Temperature-density diagram for the inner crust in the smooth composition model. The quantities $T_{\mathrm{p}}$, $E_{\text {gap }}, T_{\mathrm{U}}$, and $\tilde{T}_{\mathrm{U}}$ are described in the text. The vertical dotted line indicates the neutronization density at which the composition of the matter changes sharply, causing a weak jump in all of the parameters indicated in the figure.

The electron energy spectrum in the neutron star crust is slightly distorted by the interaction with the lattice. This interaction is strongest when the states of free electrons with momenta $\mathbf{p}=\hbar \mathbf{k}$ and $\mathbf{p}-\mathbf{g}$ have close energies. Here, $\mathbf{g}$ is a reciprocal lattice vector. We are interested in the electron states near the Fermi surface. Therefore, we will associate a set of vectors $\mathbf{p}_{0}$ of length $p_{\mathrm{F}}$ for which the condition for the energies of free electrons with momenta $\mathbf{p}_{0}$ and $\mathbf{p}_{0}-\mathbf{g}$ being equal is met with each reciprocal lattice vector $\mathbf{g}$ that does not exceed the diameter of the Fermi sphere $\left(g \leq 2 k_{\mathrm{F}}\right)$. These vectors form a circumference on the Fermi surface and are specified by the condition $\mathbf{p}_{0} \cdot \mathbf{g}=g^{2} / 2$ (see Fig. 2 ). The set of these circumferences for all vectors $\mathrm{g} \leq 2 k_{\mathrm{F}}$ forms the region of intersections between the boundaries of the Brillouin zones and the Fermi sphere. Their projections onto the plane of the faces of a bcc lattice are shown in Fig. 3. As an example, we chose matter composed of atoms with nuclear charges $Z=2,10$, 30 , and 50. The thickness of the lines corresponds (on the scale of the figure) to twice the width of the region of efficient mixing of the ultrarelativistic electron wave functions $2 \Delta k_{\text {mix }}$ calculated in the pointlike nucleus approximation by neglecting the Debye-Waller factor. The boundary of the Fermi sphere is indicated by the thick circumference; its center is marked by the point. The quantity $q_{\mathrm{BZ}}$ is indicated by the segment in the lower left corner of each figure. The bulk of 


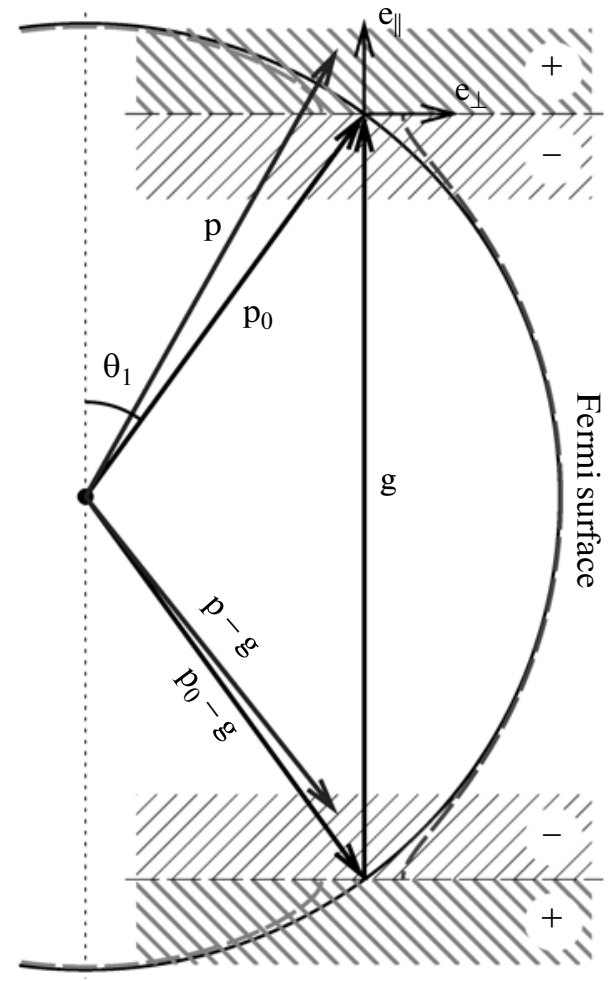

Fig. 2. Diagram showing the positions of the momenta $\mathbf{p}, \mathbf{p}-\mathbf{g}, \mathbf{p}_{0}$ and the reciprocal lattice vector $\mathbf{g}$ near the Fermi sphere in the extended zone scheme. The latter is indicated in the figure by an arc of a circumference. The thick dashed line indicates the Fermi surface with allowance made for the interaction with the lattice (it undergoes a discontinuity near the vector $\mathbf{p}_{0}$; for clarity, the gap width was increased by more than an order of magnitude compared to the typical values in the neutron star crust). The thick and thin hatching correspond, respectively, to the "+" and "-" states in the periodic zone scheme. The vertical dotted line passes through the center of the Fermi sphere marked by the point and is parallel to the vector $\mathbf{g}$.

the Fermi surface is not subjected to any significant interaction with the static lattice and remains white in Fig. 3. The electrons in these regions may be considered free with a good accuracy. Since the Brillouin zones are polyhedrons, there are points on the Fermi surface that belong to their edges. Several circumferences corresponding to different reciprocal lattice vectors $\mathbf{g}$ intersect at these points and, hence, more than two free electron wave functions are mixed in their neighborhood. However, the corresponding regions occupy a very small part of the Fermi surface and should not enhance significantly the scattering. We will neglect them when considering the electron states and will take into account only the pair mixing of the wave functions described by the momenta $\mathbf{p}$ and $\mathbf{p}-\mathbf{g}$.

Consider the electron state described in the ex-

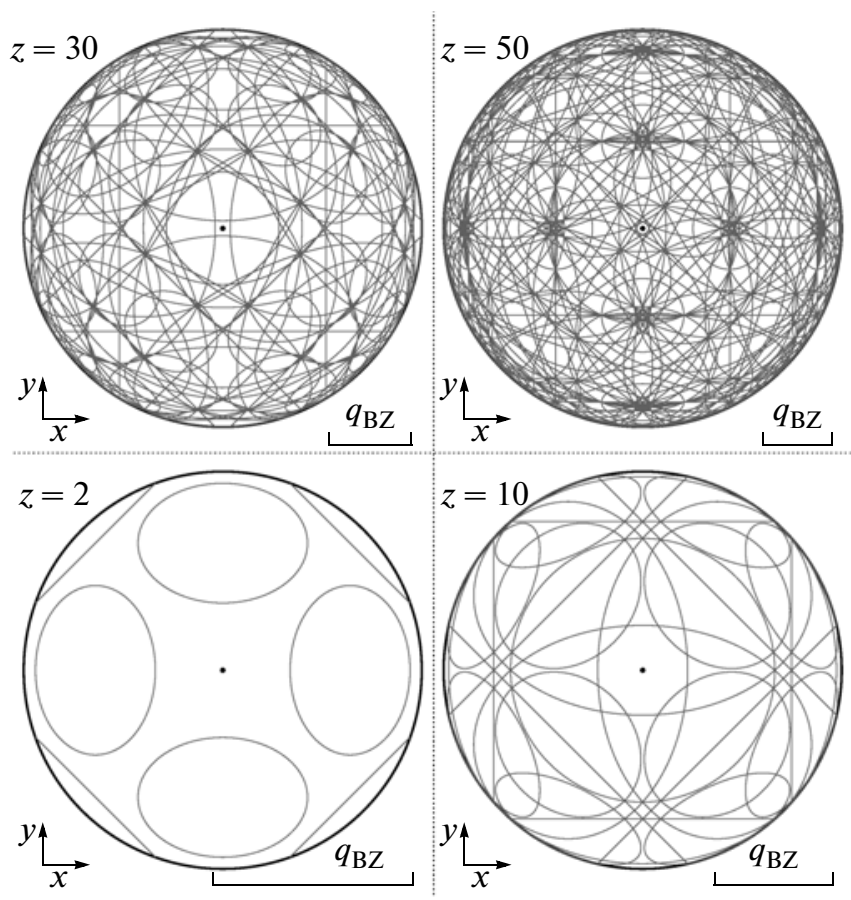

Fig. 3. Projections of the lines of intersection between the boundaries of the Brillouin zones and the Fermi sphere (large circumferences) onto the face of a bcc lattice (the $(x, y)$ plane) for charges $Z=2,10,30$, and 50 . The thickness of the lines corresponds to twice the width of the region of the greatest distortion of the electron wave functions $2 \Delta k_{\text {mix }}$. The quantity $q_{\mathrm{BZ}}$ is shown in the lower left corner of each figure; the point indicates the center of the Fermi sphere.

tended zone scheme by a quasi-momentum $\mathbf{p}$ close to one of the vectors $\mathbf{p}_{0}$ and lying in the same plane with it and the corresponding vector $\mathbf{g}$. The energy of this state unperturbed by the interaction with the lattice is specified by the expression

$$
\varepsilon_{\mathbf{k}} \approx \epsilon_{\mathrm{F}}+C_{\|} c \Delta p_{\|}+C_{\perp} c \Delta p_{\perp}
$$

where

$$
C_{\|}=\frac{g}{2 k_{\mathrm{F}}} ; \quad C_{\perp}=\sqrt{1-C_{\|}^{2}} .
$$

Here, the vector $\Delta \mathbf{p}=\mathbf{p}-\mathbf{p}_{0}$. The quantities $\Delta p_{\|}$ and $\Delta p_{\perp}$ are the coordinates of the vector $\Delta \mathbf{p}$ in a Cartesian coordinate system with such unit vectors $\mathbf{e}_{\|}$and $\mathbf{e}_{\perp}$ that $\mathbf{p}_{0}=p_{\mathrm{F}}\left(C_{\|} \mathbf{e}_{\|}+C_{\perp} \mathbf{e}_{\perp}\right)$ (see Fig. 2). The projection of $\Delta \mathbf{p}$ onto the $\mathbf{e}_{\varphi}=\left[\mathbf{e}_{\|} \times \mathbf{e}_{\perp}\right]$ axis is equal to zero in view of the choice of vector $\mathbf{p}_{0}$.

Since we consider the state with a quasi-momentum $\mathbf{p}$ close to $\mathbf{p}_{0}$, it will be mixed most strongly with the state $\mathbf{p}-\mathbf{g}$, while we will neglect the mixing with other states. Therefore, in the periodic zone scheme, from a generally infinite set of states corresponding to each quasi-momentum $\mathbf{p}$, we will choose two states 
that correspond to the quasi-momenta $\mathbf{p}$ and $\mathbf{p}-\mathbf{g}$ in the extended zone scheme. We will denote the states with higher and lower energies, respectively, by the indices "+" and "--." The ions in the neutron star crust are completely ionized; therefore, the electronatomic nucleus interaction potential $U(r)$ may be considered to be known. Since the electron Fermi energy is great compared to the interaction energy of electrons with atomic nuclei, the energy of the chosen pair of electron states can be calculated using the perturbation theory (see, e.g., Ziman 1960):

$$
\begin{aligned}
E_{\mathbf{k}}^{ \pm} & =\frac{\varepsilon_{\mathbf{k}}+\varepsilon_{\mathbf{k}-\mathbf{g}}}{2} \pm \sqrt{\left(\frac{\varepsilon_{\mathbf{k}}-\varepsilon_{\mathbf{k}-\mathbf{g}}}{2}\right)^{2}+E_{\mathbf{g}}^{2}} \\
& =\epsilon_{\mathrm{F}}+C_{\perp} c \Delta p_{\perp} \pm \sqrt{\left(C_{\|} c \Delta p_{\|}\right)^{2}+E_{\mathbf{g}}^{2}},
\end{aligned}
$$

where

$$
E_{\mathbf{g}}=n_{\mathrm{i}} U_{\mathbf{g}}=4 \pi n_{\mathrm{i}} Z e^{2} \phi(g)
$$

is the gap half-width, $U_{\mathbf{g}}=4 \pi Z e^{2} \phi(g)$ is the Fourier component of the potential $U(r)$, and

$$
\varepsilon_{\mathbf{k}-\mathbf{g}}=\epsilon_{\mathrm{F}}-C_{\|} \Delta p_{\|}+C_{\perp} \Delta p_{\perp}
$$

is the unperturbed energy of the state with momentum $\mathbf{p}-\mathbf{g}$ (see Fig. 2). The quantity $\phi(\mathbf{g})=\phi(g)$ does not depend on the direction of $\mathbf{g}$, because the potential $U(r)$ is isotropic. It can be represented as (see, e.g., Gnedin et al. 2001)

$$
\phi(q)=\mathrm{e}^{-W(q)} \frac{F(q)}{q^{2} \epsilon(q)} .
$$

Here,

$$
F(q)=\frac{1}{Z} \int e n_{\mathrm{p}}(r) \exp (\imath \mathbf{r} \cdot \mathbf{q}) \mathrm{d}^{3} r
$$

is the form factor of the atomic nuclei that describes the electric charge (proton) distribution over the nucleus, $W(q)$ is the Debye-Waller factor that allows for the ion vibrations about the equilibrium position, and $\epsilon(q)$ is the static longitudinal dielectric function of the electron plasma. In the pointlike nucleus approximation $\left[n_{p}=Z \delta(\mathbf{r})\right]$ used in Fig. $3, F(q)=1$. To calculate the dielectric function, we will use the simplest Thomas-Fermi model

$$
\epsilon(q)=1+\frac{k_{\mathrm{TF}}^{2}}{q^{2}},
$$

in which the screening of the test charge potential by electrons is characterized by one parameter-the Thomas-Fermi wave number

$$
k_{\mathrm{TF}}^{2}=4 \pi^{2} e^{2} \frac{\partial n_{e}}{\partial \mu_{\mathrm{e}}} \approx \frac{\alpha_{\mathrm{f}}}{\pi}\left(2 k_{\mathrm{F}}\right)^{2} .
$$

Here, $\mu_{\mathrm{e}} \approx \epsilon_{\mathrm{F}}$ is the electron chemical potential and $\alpha_{\mathrm{f}} \equiv e^{2} /(\hbar c) \approx 1 / 137$ is the fine-structure constant.
This model is applicable at $q \ll k_{\mathrm{TF}}$. However, we will also be interested in the regions corresponding to wave numbers $q \sim k_{\mathrm{F}}$, in which, strictly speaking, a more accurate theory should be used. For the case of free degenerate electrons, the dielectric function was calculated by Jancovici (1962). However, he disregarded the distortion of the electron energy spectrum by the interaction with the lattice. ${ }^{1}$ It can be important for screening at wave vectors $\mathbf{q}$ close to the reciprocal lattice vectors and can lead to an anisotropy of the dielectric function. Allowance for the latter effect seems an interesting subject matter for a separate study that, to the best of my knowledge, has not yet been carried out for the case of neutron star crusts. Nevertheless, at large transferred momenta $q \sim k_{\mathrm{F}}$, the screening is weak $(\epsilon \approx 1)$. Therefore, when calculating the electrical conductivity, applying an accurate screening model should not lead to qualitatively new effects compared to the ThomasFermi model. This allows us to restrict ourselves to using the latter in this paper. In the periodic zone scheme, the wave function with allowance made for the interaction with the static crystal lattice can be written as

$$
\begin{gathered}
\Psi_{\sigma, \mathbf{p}}^{+}=u_{\mathbf{k}} \Psi_{\sigma}^{\text {free }}(\mathbf{p})+v_{\mathbf{k}} \Psi_{\sigma}^{\text {free }}(\mathbf{p}-\hbar \mathbf{g}), \\
\Psi_{\sigma, \mathbf{p}}^{-}=v_{\mathbf{k}} \Psi_{\sigma}^{\text {free }}(\mathbf{p})-u_{\mathbf{k}} \Psi_{\sigma}^{\text {free }}(\mathbf{p}-\hbar \mathbf{g}),
\end{gathered}
$$

where $\Psi_{\sigma}^{\text {free }}(\mathbf{p})$ is the wave function of a free electron with momentum $\mathbf{p}$ and helicity $\sigma$ (Berestetskii et al. 1982) and the expansion coefficients $u_{\mathbf{k}}=$ $E_{\mathbf{g}} /\left[2 \epsilon_{\mathbf{k}}\left(\epsilon_{\mathbf{k}}-\xi_{\mathbf{k}}\right)\right]^{1 / 2}$ and $v_{\mathbf{k}}=\left(\epsilon_{\mathbf{k}}-\xi_{\mathbf{k}}\right)^{1 / 2} /\left(2 \epsilon_{\mathbf{k}}\right)^{1 / 2}$. Here, $\quad \xi_{\mathrm{k}}=C_{\|} c \Delta p_{\|} \quad$ and $\quad \epsilon_{\mathbf{k}}=$ $=\sqrt{\left(C_{\|} c \Delta p_{\|}\right)^{2}+E_{\mathbf{g}}^{2}}$.

The correspondence between the state indices and quasi-momenta in the extended and periodic zone schemes is given in the table (see also Fig. 2). For example, at $\Delta p_{\|}<0$, the state with quasi-momentum $\mathbf{p}$ and the "+" index in the periodic zone scheme corresponds to the state with quasi-momentum $\mathbf{p}-\mathbf{g}$ in the extended zone scheme.

Following Gnedin et al. (2001), let us estimate the temperatures at which the distortion of the wave functions and the electron dispersion relation by the interaction with the static lattice becomes significant for the consideration of electron-phonon scattering. We will estimate the characteristic gap width at $g \sim k_{\mathrm{F}}$ :

$$
E_{\text {gap }}=2 E_{g=k_{\mathrm{F}}} \sim \frac{4 e^{2}}{3 \pi} \frac{F\left(k_{\mathrm{F}}\right) \mathrm{e}^{-W\left(k_{\mathrm{F}}\right)}}{\epsilon\left(k_{\mathrm{F}}\right)} k_{\mathrm{F}} .
$$

\footnotetext{
${ }^{1}$ Taking an opportunity, I express may gratitude to one of the anonymous referees who drew may attention to this effect.
} 
Table of correspondence between the state parameters in the periodic and extended zone schemes

\begin{tabular}{c|c|c}
\hline Parameter & “+” State & “-” State \\
\hline$\Delta p_{\|}>0$ & $\mathbf{p}$ & $\mathbf{p}-\mathbf{g}$ \\
$\Delta p_{\|}<0$ & $\mathbf{p}-\mathbf{g}$ & $\mathbf{p}$ \\
\hline
\end{tabular}

Note. The quasi-momentum describing the state in the extended Brillouin zone scheme that corresponds to a given quasimomentum $\mathbf{p}$ and state index ("+" or "-") in the periodic zone scheme is specified. The rows correspond to the opposite signs of the components $\Delta p_{\|}$.

The numerical value of the gap half-width (in temperature units) is indicated in Fig. 1 by the solid line. Because of the difference between the phonon and electron velocities, the presence of gaps begins to affect significantly the electron-phonon scattering at an order of magnitude lower temperatures, $T \lesssim$ $\lesssim T_{\mathrm{U}}$. Indeed, the interaction of electrons with the static lattice distorts their dispersion relation in regions with a characteristic size $\Delta k_{\text {mix }} \sim E_{\text {gap }} / V_{\mathrm{e}}$, where $V_{\mathrm{e}} \approx c$ is the electron velocity, whereas at $k_{\mathrm{B}} T \sim E_{\text {gap }}$ the electrons are efficiently scattered by phonons with wave numbers $q \sim k_{\mathrm{B}} T / V_{\mathrm{ph}}$, where $V_{\mathrm{ph}} \sim \omega_{p} / q_{\mathrm{BZ}}$ is a typical velocity of acoustic phonons $\left(V_{\mathrm{ph}} \ll c\right)$. Accordingly, electrons with momenta lying in the region with a characteristic size $q \sim$ $\left(c / V_{\text {ph }}\right) \Delta k_{\text {mix }} \gg \Delta k_{\text {mix }}$ are involved in the scattering. In the bulk of this region, the electron states are distorted only slightly. Therefore, at $k_{\mathrm{B}} T \sim E_{\text {gap }}$, the bulk of the phonon-electron scattering events may be considered in the approximation of free electrons, as was done by Potekhin et al. (1999). However, as the temperature decreases, the characteristic phonon wave number $q$ becomes comparable to $\Delta k_{\text {mix }}$ and the approximation of free electrons becomes inapplicable for the consideration of electron-phonon scattering. A more detailed description of the electron states is required. This occurs at temperatures

$$
\begin{gathered}
T \lesssim T_{\mathrm{U}}=\frac{V_{\mathrm{ph}}}{V_{\mathrm{e}}} \frac{E_{\text {gap }}}{k_{\mathrm{B}}} \\
\sim \alpha_{\mathrm{f}} T_{\mathrm{p}} \frac{Z^{1 / 3}}{3} \mathrm{e}^{-W\left(k_{\mathrm{F}}\right)} F\left(k_{\mathrm{F}}\right) .
\end{gathered}
$$

In this estimate, we neglected the electron screening (because $k_{\mathrm{TF}} \ll k_{\mathrm{F}}$ ). We will call the characteristic temperature $T_{\mathrm{U}}$ the freezing temperature of Umklapp processes. By the standards of neutron stars, it is low (see the dash-dotted line in Fig. 1) but is reached, for example, in isolated neutron stars with an age $t \gtrsim 10^{6}$ yr (see, e.g., Gusakov et al. 2005;
Yakovlev et al. 2011; Shternin et al. 2011), which can manifest themselves as pulsars (Lorimer 2010; Popov et al. 2010; Boldin and Popov 2010). Note that Gnedin et al. (2001) estimated the freezing temperature of Umklapp processes in the approximation of $F(q)=1: \widetilde{T}_{\mathrm{U}}=\alpha_{\mathrm{f}} T_{\mathrm{p}} Z^{1 / 3} / 3$. However, $F\left(k_{\mathrm{F}}\right) \sim 0.3$ near the boundary with the stellar core. For this reason, the temperature $T_{\mathrm{U}}$ differs from $\tilde{T}_{\mathrm{U}}$, which is indicated in Fig. 1 by the dashed line.

\section{ELECTRICAL CONDUCTIVITY. GENERAL FORMULAS}

Once the matter parameters and the electron states in the neutron star crust have been described, we can turn to calculating the electrical conductivity. Directly solving the kinetic equation by taking into account all scattering processes is a very complex problem. We will restrict ourselves to an estimation using the variational method (see, e.g., Ziman 1960) and will calculate the electrical conductivity as the maximum of the functional

$$
\sigma=\left|\mathbf{I}_{1}\right|^{2} / I_{2},
$$

when varying the trial function $\Phi_{\mathbf{k}}$ that describes the deviation of the electron distribution function from the equilibrium one. Here, the integral

$$
\begin{gathered}
I_{2}=\frac{1}{k_{\mathrm{B}} T} \sum_{\nu} \iiint\left(\Phi_{\mathbf{k}}-\Phi_{\mathbf{k}^{\prime}}\right)^{2} P_{\mathbf{p}, \mathbf{q}, \nu}^{\mathbf{p}^{\prime}} \\
\times \frac{\mathrm{d}^{3} k}{(2 \pi)^{3}} \frac{\mathrm{d}^{3} k^{\prime}}{(2 \pi)^{3}} \frac{\mathrm{d}^{3} q}{(2 \pi)^{3}}
\end{gathered}
$$

specifies twice the entropy generation rate during the absorption of phonons (which is equal to the total entropy generation rate in the phonon absorption and production processes). The integration is over the electron quasi-momenta (in the extended zone scheme) before and after scattering $\left(\mathbf{p}=\hbar \mathbf{k}\right.$ and $\mathbf{p}^{\prime}=$ $\left.\hbar \mathbf{k}^{\prime}\right)$ as well as over the wave vector of the scattering (absorbed) phonon q. The corresponding scattering probability $P_{\mathbf{p}, \mathbf{q}, \nu}^{\mathbf{p}^{\prime}}$ in the limit $\mathbf{p}^{\prime} \approx \mathbf{p}-\mathbf{g}$ of interest to us is calculated in Appendix A. The summation is over the branches of phonon modes $\nu$. Since the electron scattering occurs with the conservation of helicity ( see Appendix A), the corresponding indices do not affect the result and are omitted here. The normalization integral $I_{1}$ in (17) is given by the expression

$$
\mathbf{I}_{1}=\int e \mathbf{V}_{\mathbf{k}} \Phi_{\mathbf{k}} \frac{\partial f}{\partial E_{\mathbf{p}}} \frac{\mathrm{d}^{3} k}{(2 \pi)^{3}},
$$

where $f=f(\mathbf{p})=1 /\left[\exp \left(\left(E_{\mathbf{p}}-\mu_{\mathrm{e}}\right) / k_{\mathrm{B}} T\right)+1\right]$ is the equilibrium Fermi electron distribution function. 
Let us choose a simple trial function (for the properness of this choice, see Appendix B) that does not contain the variational parameters $\Phi_{\mathbf{k}}=\mathbf{V}_{\mathbf{k}} \cdot \mathbf{u}$, where $\mathbf{V}_{\mathbf{k}}=\partial E_{\mathbf{k}} / \partial \mathbf{p}$ is the electron velocity and $\mathbf{u}$ is a unit vector in the electric field direction. The "+" or "-" state index is not required here, because we use the extended zone scheme, where the quasimomentum uniquely specifies the electron state. It can be shown that this trial function gives a better estimate of the electrical conductivity than the yet simpler trial function $\Phi_{\mathbf{k}}=\mathbf{k} \cdot \mathbf{u} / k$. The integral $\mathbf{I}_{1}$ can be calculated in the approximation of free electrons $\left(\mathbf{V}_{\mathbf{k}}=\mathbf{n} c\right.$, where $\left.\mathbf{n}=\mathbf{k} / k\right)$, because the electrons are almost free on the bulk of the Fermi surface (see Fig. 3). Simple integration gives $\left|\mathbf{I}_{1}\right|=$ $e c k_{\mathrm{F}}^{2} /\left(6 \pi^{2} \hbar\right)$. After the substitution of the scattering probability $P_{\mathbf{p}, \mathbf{q}, \nu}^{\mathbf{p}^{\prime}}$ (Eq. (A6)), the integration over $\mathbf{k}^{\prime}$ is performed easily using a delta function of the quasimomentum. Recall that we consider the electrical conductivity at low temperatures, when the states with quasi-momenta $\mathbf{p}$ lying near the vectors $\mathbf{p}_{0}$ make a major contribution to the electron-phonon scattering (see Fig. 2). Describing these states in the periodic zone scheme, we will write the integral over the electron states in (18) as the sum of integrals over the two-dimensional vectors $\Delta \mathbf{k}=\Delta \mathbf{p} / \hbar=(\mathbf{p}-$ $\left.\mathbf{p}_{0}\right) / \hbar$ and the azimuthal angle $\varphi_{\mathbf{k}}$ of the vector $\mathbf{k}$ :

$$
\begin{gathered}
I_{2}=\frac{n_{\mathrm{i}}}{4(2 \pi)^{5} m_{\mathrm{i}} k_{\mathrm{B}} T} \sum_{(j)} \sum_{\left(j^{\prime}\right)} \sum_{\mathbf{g}} \sum_{\nu} \\
\times \int\left[\left(\mathbf{V}_{\mathbf{k}}-\mathbf{V}_{\mathbf{k}^{\prime}}\right) \cdot \mathbf{u}\right]^{2} \frac{n_{\mathbf{q}, \nu}}{\omega_{\mathbf{q}, \nu}} f_{\mathbf{p}}\left(1-f_{\mathbf{p}^{\prime}}\right) \\
\times\left\{\left(\mathbf{e}_{\mathbf{q}, \nu} \cdot \mathbf{g}\right) U_{\mathbf{g}} C_{\perp} F_{u v}^{\mathbf{g}}\right\}^{2} \\
\times \delta\left(E_{\mathbf{k}}+\hbar \omega_{\mathbf{q}, \nu}-E_{\mathbf{k}^{\prime}}\right) C_{\perp} k_{\mathrm{F}} \mathrm{d} \varphi_{\mathbf{k}} \mathrm{d}^{2} \Delta k \mathrm{~d}^{3} q .
\end{gathered}
$$

The summation is over the vectors $\mathbf{g}$ and the electron state indices $(j),\left(j^{\prime}\right)=$ ",+ " "-." In the integrand, the mode indices $(j)$ and $\left(j^{\prime}\right)$ are omitted to save space. The factors $F_{u v}^{\mathbf{g}}$ are determined in Appendix A. The additional coefficient $1 / 2$ in (20) is necessary, because during the summation over the index $(j)$, each electron state before scattering is taken into account twice due to the redundancy of the periodic zone scheme.

Note that for the vectors $\mathbf{g}$, which can be obtained from each other by the lattice symmetry transformations, the integral in (20) will differ only by the factor $\left[\left(\mathbf{V}_{\mathbf{k}}-\mathbf{V}_{\mathbf{k}^{\prime}}\right) \cdot \mathbf{u}\right]^{2}$ that does not satisfy this symmetry due to the presence of vector $\mathbf{u}$. Since the summation in (20) is over the reciprocal lattice vectors, for each of them we can perform averaging under the integral over, in general, 48 reciprocal lattice vectors $\mathbf{g}_{\mathrm{e}}$ that are obtained from a given vector $\mathbf{g}$ by the cubic lattice symmetry transformations (the permutation and reversal of coordinates)

$$
\frac{1}{48} \sum_{\mathbf{g}_{\mathrm{e}}}\left[\left(\mathbf{V}_{\mathbf{k}}-\mathbf{V}_{\mathbf{k}^{\prime}}\right) \cdot \mathbf{u}\right]^{2}=\frac{1}{3}\left(\mathbf{V}_{\mathbf{k}}-\mathbf{V}_{\mathbf{k}^{\prime}}\right)^{2} .
$$

This assertion is proven in Appendix C.

The vector $\Delta \mathbf{k}$ in the local Cartesian coordinate system $\left(\mathbf{e}_{\|}, \mathbf{e}_{\perp}\right)$ can be written as $\left(\Delta k_{\|}, \Delta k_{\perp}\right)$. The velocity difference $\mathbf{V}_{\mathbf{k}}-\mathbf{V}_{\mathbf{k}^{\prime}}=\partial \Delta E / \partial \mathbf{p}$, where the energy difference

$$
\begin{gathered}
\Delta E=E_{\mathbf{k}}^{(j)}-E_{\mathbf{k}^{\prime}}^{\left(j^{\prime}\right)}=-C_{\perp} c \hbar q_{\perp} \\
\pm \sqrt{\left(C_{\|} c \hbar \Delta k_{\|}\right)^{2}+E_{\mathbf{g}}^{2}} \\
\mp \sqrt{\left[C_{\|} c \hbar\left(\Delta k_{\|}+q_{\|}\right)\right]^{2}+E_{\mathbf{g}}^{2}} .
\end{gathered}
$$

Here, the first "+" or "-" sign corresponds to the index $(j)$, while the second "-" or "+" sign corresponds to the index $\left(j^{\prime}\right)$ taken with the opposite sign. It is easy to verify that the velocity difference $\mathbf{V}_{\mathbf{k}}-\mathbf{V}_{\mathbf{k}^{\prime}}$ does not depend on $\Delta k_{\perp}$ and is directed along the vector $\mathbf{g}$. This allowed the integration to be performed over the component $\Delta k_{\perp}$ :

$$
\begin{gathered}
\int f(E)\left[1-f\left(E+\hbar \omega_{\mathbf{q}, \nu}\right)\right] \mathrm{d} \Delta k_{\perp} \\
=\frac{\omega_{\mathbf{q}, \nu}}{C_{\perp} \hbar c} \frac{1}{1-e^{-z_{\nu}}},
\end{gathered}
$$

where $z_{\nu}=\hbar \omega_{\mathbf{q}, \nu} /\left(k_{\mathrm{B}} T\right)$ and the relation $\mathrm{d} E_{\mathbf{k}}^{(j)}=$ $C_{\perp} \hbar c \mathrm{~d} \Delta k_{\perp}$ (see Eq. (7)) is used. After the change of variables $\mathrm{d} \Delta E=\mathrm{d}\left(E_{\mathbf{k}}-E_{\mathbf{k}^{\prime}}\right)=\hbar\left|\mathbf{V}_{\mathbf{k}}-\mathbf{V}_{\mathbf{k}^{\prime}}\right| \mathrm{d} \Delta k_{\|}$, the integration over $\mathrm{d} \Delta E$ is performed easily using a delta function of the energy. As a result, the integral $I_{2}$ can be written as

$$
I_{2}=\frac{n_{\mathrm{i}}}{12(2 \pi)^{5}} \frac{p_{\mathrm{F}}}{\hbar^{2} m_{\mathrm{i}} k_{\mathrm{B}} T} \sum_{\mathbf{g}} U^{2}(\mathbf{g}) \sum_{\nu} I_{3}^{\mathbf{g}, \nu},
$$

where we introduced a new integral

$$
\begin{aligned}
& I_{3}^{\mathbf{g}}=\frac{C_{\perp}^{2}}{c} \sum_{(j)} \sum_{\left(j^{\prime}\right)} \sum_{\nu} \int\left|\mathbf{V}_{\mathbf{k}}-\mathbf{V}_{\mathbf{k}^{\prime}}\right| \\
& \times \frac{n_{\mathbf{q}, \nu}}{1-e^{-z_{\nu}}}\left(F_{u v}^{\mathbf{g}}\right)^{2}\left(\mathbf{e}_{\mathbf{q}, \nu} \cdot \mathbf{g}\right)^{2} \mathrm{~d} \varphi_{\mathbf{k}} \mathrm{d}^{3} q .
\end{aligned}
$$

The electrical conductivity can now be easily represented via the effective electron relaxation time $\tau$ :

$$
\sigma=\frac{e^{2} n_{e} \tau}{x_{\mathrm{r}} m_{e}}, \quad \tau=\frac{p_{\mathrm{F}}^{2} v_{\mathrm{F}}}{4 \pi Z^{2} e^{4} n_{i} \Lambda_{\sigma}},
$$

where

$$
\Lambda_{\sigma}=\frac{p_{\mathrm{F}}}{8 \pi^{2}} \frac{\hbar}{k_{\mathrm{B}} T m_{\mathrm{i}}} \sum_{\mathbf{g}} \phi(g)^{2} I_{3}^{\mathbf{g}}
$$




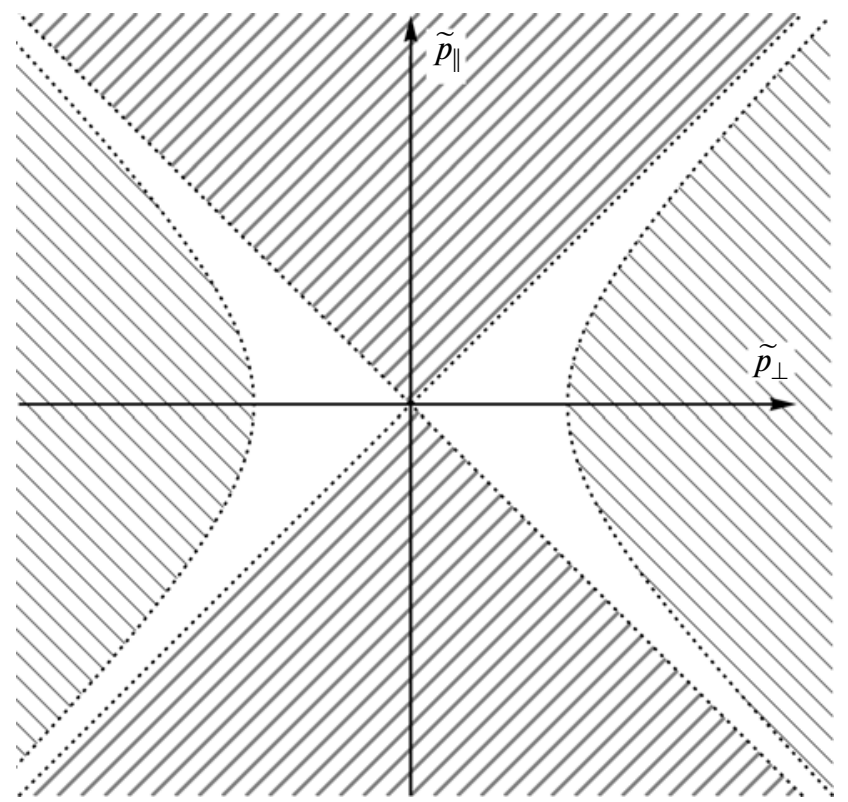

Fig. 4. Phonon wave functions corresponding to different types of scattering processes. The thick hatching indicates the "++" and "--" processes; the thin hatching indicates the "+-" and "-+" processes. The integration in (30) is over all hatched regions.

may be considered as a "generalized" Coulomb logarithm. Since the phonon velocities are low compared to the electron ones, we may neglect the phonon energy in the energy conservation law and write the quasi-momenta of the electrons capable of being involved in the scattering by a phonon with a wave number $\mathbf{q}$ as

$$
\begin{gathered}
\Delta p_{\|}=-\frac{\widetilde{p}_{\|}}{2} \pm \frac{\widetilde{p}_{\perp}}{2} \sqrt{1+4 \frac{\widetilde{p}_{\mathbf{g}}^{2}}{\Delta \widetilde{p}^{2}}} \\
\Delta p_{\| \mid}^{\prime}=\frac{\widetilde{p}_{\|}}{2} \pm \frac{\widetilde{p}_{\perp}}{2} \sqrt{1+4 \frac{\widetilde{p}_{\mathbf{g}}^{2}}{\Delta \widetilde{p}^{2}}} .
\end{gathered}
$$

Here, we use the notation $\widetilde{p}_{\mathbf{g}}=E_{\mathbf{g}} /\left(c C_{\|}\right), \widetilde{p}_{\perp}=$ $\hbar q_{\perp} C_{\perp} / C_{\|}, \widetilde{p}_{\|}=\hbar q_{\|}, \Delta \widetilde{p}^{2}=\widetilde{p}_{\|}^{2}-\widetilde{p}_{\perp}^{2}$. Substituting the explicit form of the functions $F_{u v}^{\mathbf{g}}$ for electrons with quasi-momenta (28) and (29), we will make analytical transformations and write a general expression for the integral

$$
\begin{gathered}
I_{3}^{\mathbf{g}}=2 C_{\|} C_{\perp}^{2} \\
\times \sum_{\nu} \int \frac{\left(\Delta \widetilde{p}^{2}\right)^{7 / 2}\left(\Delta \widetilde{p}^{2}+4 \widetilde{p}_{\mathbf{g}}^{2}\right)^{1 / 2}}{\left(4 \widetilde{p}_{\|}^{2} \widetilde{p}_{\mathbf{g}}^{2}+\left(\Delta \widetilde{p}^{2}\right)^{2}\right)^{2}} \\
\times \frac{e^{-z_{\nu}}}{\left(1-e^{-z_{\nu}}\right)^{2}}\left(\mathbf{e}_{\mathbf{q}, \nu} \cdot \mathbf{g}\right)^{2} \mathrm{~d} \varphi_{\mathbf{k}} \mathrm{d}^{3} q .
\end{gathered}
$$

Here, the integration is over the region where either $\Delta \widetilde{p}^{2}>0$ or $\Delta \widetilde{p}^{2}<-4 \widetilde{p}_{\mathbf{g}}^{2}$ (the hatched region in Fig. 4) in which the conservation laws admit scattering.

In Eq. (30), the summation over the electron state indices $(j)$ and $\left(j^{\prime}\right)$ has already been performed. However, to understand the electronphonon scattering physics at low temperatures, it is important to know the transitions between what electron states are realized at a given value of the vector q. We will denote the types of scattering processes by indices of the form " \pm \pm ," where the first and second signs are specified by the initial, $(j)$, and final, $\left(j^{\prime}\right)$, electron state indices, respectively. For example, the " ++ " process is the transition from state $\Psi_{\sigma, \mathbf{p}}^{+}$to $\Psi_{\sigma, \mathbf{p}^{\prime}}^{+}$(recall that the helicity $\sigma$ is conserved during scattering). It can be shown that the "++" and "--" processes take place at $\left|\widetilde{p}_{\|}\right|>\left|\widetilde{p}_{\perp}\right|$ (the thick hatching in Fig. 4), while the "+-" and "-+" processes take place at $\widetilde{p}_{\perp}^{2}>$ $>\widetilde{p}_{\|}^{2}+4 \widetilde{p}_{\mathbf{g}}^{2}$ (the thin hatching in Fig. 4). No phonons with a wave vector lying in the region $\widetilde{p}_{\|}^{2}<\widetilde{p}_{\perp}^{2}<$ $<\widetilde{p}_{\|}^{2}+4 \widetilde{p}_{\mathbf{g}}^{2}$ (the unhatched region in Fig. 4) are involved in the electron scattering processes corresponding to the vector $\mathbf{g}$, because this scattering cannot satisfy the energy and quasi-momentum conservation laws (see Eqs. (28) and (29)). Nevertheless, they can be involved in the scattering for a different orientation of the reciprocal lattice vector and in the normal electron scattering processes considered by Raikh and Yakovlev (1982) and occurring on the entire Fermi surface. However, the latter do not contribute significantly to the electrical conductivity (see Section 7).

As we see from Fig. 4, phonons with an arbitrarily small wave number $q$ can be involved in the electron scattering in the "++" and "-- " processes. However, following Raikh and Yakovlev (1982) and by analogy with many terrestrial materials (see, e.g., Ziman 1960), Gnedin et al. (2001) suggested that for a phonon to be involved in the Umklapp process, it must have a wave number no smaller than some critical value $q_{\min }$. This value was estimated as the width of the regions perturbed by the interaction with the lattice, $q_{\min } \sim k_{\min } \sim E_{\mathrm{g}} /(\hbar c)$. It was on the basis of this erroneous estimate that the model of exponential reduction of the electron scattering rate in Umklapp processes at low temperatures $T \lesssim T_{\mathrm{U}}$ (see Eq. (40)) was proposed. In reality, $q_{\min }$ is determined by the minimum distance from the Fermi surface to the boundary of the Brillouin zone. For many terrestrial conductors, it can be nonzero due to the small number of free electrons per atom (see, e.g., Ziman 1960). Accordingly, such materials will 


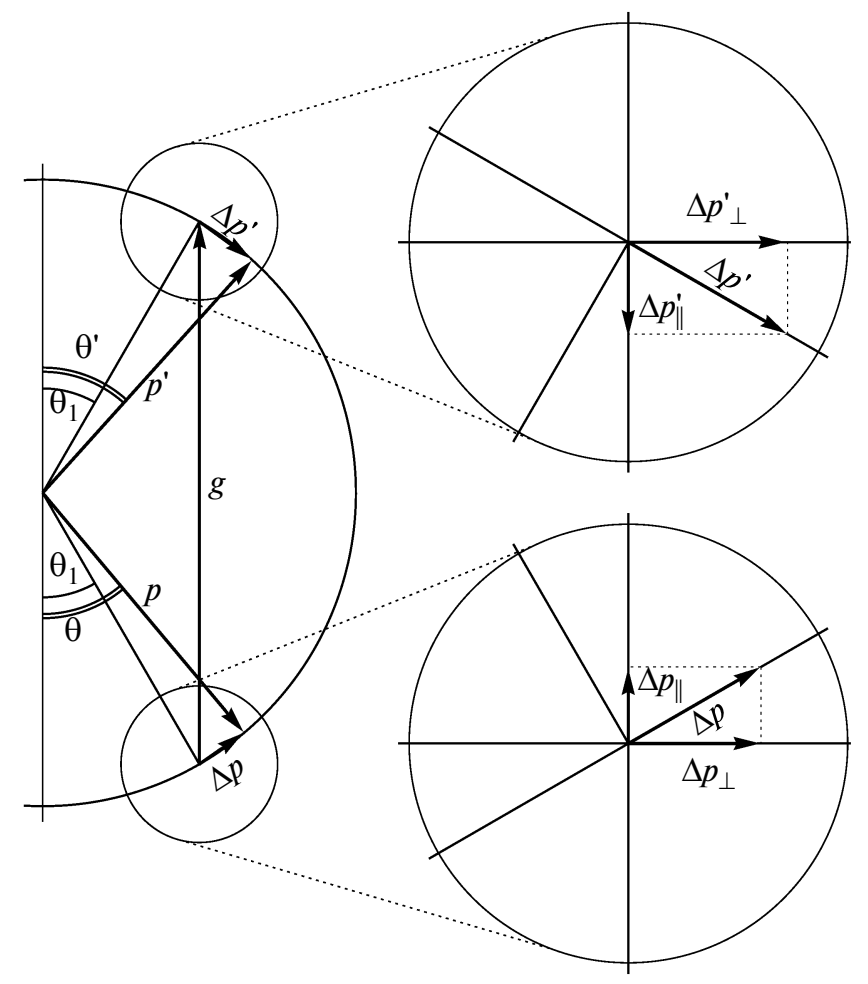

Fig. 5. Diagram showing the positions of the electron momentum on the Fermi surface before and after scattering.

be subjected to exponential freezing of Umklapp processes at low temperatures. However, as has already been noted above, there are many electrons per crystal lattice site in the neutron star crust and the Fermi surface intersects with the boundaries of the Brillouin zones. As a result, phonons with arbitrarily small wave numbers can be involved in Umklapp processes and, hence, the exponential freezing model is inapplicable to the scattering rates. A more detailed analysis performed here is required.

Note that the "+-" and "-+" processes actually require the involvement of phonons with wave numbers $q \gtrsim q_{\min } \approx k_{\min }$ (see Fig. 4 ). Therefore, this type of scattering is subjected to exponential freezing (of the scattering rate $\propto \exp \left(-T_{\mathrm{U}} / T\right)$ at $\left.T \ll T_{\mathrm{U}}\right)$ and is insignificant at low temperatures.

\section{THE HIGH-TEMPERATURE LIMIT}

Let us show that in the case of high temperatures $T \gg T_{\mathrm{U}}$ where the electron-phonon scattering is efficient far from the boundaries of the Brillouin zones and the distortion of the electron spectrum by the interaction with the static lattice may be neglected, our result (Eqs. (27) and (30)) coincides with the well-known Coulomb logarithm obtained in the approximation of free electrons (see, e.g., Flowers and Itoh 1976; Raikh and Yakovlev 1982; Baiko and Yakovlev 1995). Let us set $\widetilde{p}_{\mathbf{g}}=0$ in (30). The integral $I_{3}^{\mathrm{g}, \nu}$ can then be rewritten as

$$
I_{3}^{\mathbf{g}}=2 C_{\|} C_{\perp}^{2} \sum_{\nu} \int \frac{e^{-z_{\nu}}\left(\mathbf{e}_{\mathbf{q}, \nu} \cdot \mathbf{g}\right)^{2}}{\left(1-e^{-z_{\nu}}\right)^{2}} \mathrm{~d} \varphi_{\mathbf{k}} \mathrm{d}^{3} q .
$$

Now, it will suffice to pass from the integration over the phonon wave vectors $\mathbf{q}$ to the integration over the positions of the electron quasi-momentum before and after scattering on the Fermi surface, which is commonly used in calculations in the approximation of free electrons. For this purpose, in accordance with Eqs. (28) and (29), we will write the longitudinal electron quasi-momentum components before and after scattering as $\Delta p_{\|}=-\widetilde{p}_{\|} / 2+\widetilde{p}_{\perp} / 2$ and $\Delta p_{\|}^{\prime}=$ $\widetilde{p}_{\|} / 2+\widetilde{p}_{\perp} / 2$ and will take $\Delta p_{\perp}=C_{\|} \Delta p_{\|} / C_{\perp}$ and $\Delta p_{\perp}^{\prime}=-C_{\|} \Delta p_{\|}^{\prime} / C_{\perp}$ as the transverse components. Obviously, the states with such quasi-momenta lie on the Fermi surface (to be more precise, on the plane tangential to it, see Fig. 5). Note that $\mathrm{d} q_{\varphi}=$ $C_{\perp} k_{\mathrm{F}}\left(\mathrm{d} \varphi_{\mathbf{k}^{\prime}}-\mathrm{d} \varphi_{\mathbf{k}}\right)$, where $\varphi_{\mathbf{k}^{\prime}}$ is the azimuthal angle of the vector $\mathbf{k}^{\prime}$. We can now pass to the integration in (31) over $\mathrm{d} \Omega$ and $\mathrm{d} \Omega^{\prime}$, the elements of the spherical angles in the directions of the vectors $\mathbf{k}$ and $\mathbf{k}^{\prime}$, and 


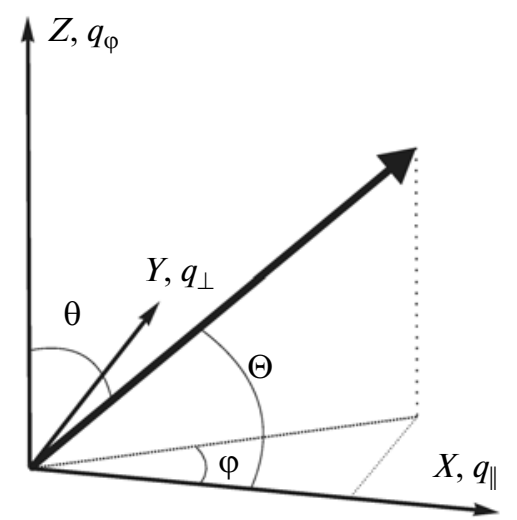

Fig. 6. Diagram showing the positions the angles in the spherical coordinate system specifying the vector $\mathbf{q}$.

white the Coulomb logarithm as

$$
\begin{gathered}
\Lambda_{\sigma}=\frac{2 p_{\mathrm{F}}^{2}}{k_{\mathrm{B}} T m_{\mathrm{i}}} \sum_{\mathbf{g}} \phi(g)^{2} g^{2} \\
\times \sum_{\nu} \int \frac{e^{-z_{\nu}}}{\left(1-e^{-z_{\nu}}\right)^{2}}\left(\mathbf{e}_{\mathbf{q}, \nu} \cdot \mathbf{g}\right)^{2}\left(1-\frac{g^{2}}{4 k_{\mathrm{F}}^{2}}\right) \frac{\mathrm{d} \Omega}{4 \pi} \frac{\mathrm{d} \Omega^{\prime}}{4 \pi} .
\end{gathered}
$$

It is easy to verify that the scattering rate defined by Eq. (32) coincides with the low-temperature asymptotics of Eqs. (3) and (4) from Raikh and Yakovlev (1982) obtained in the approximation of free electrons.

\section{THE LOW-TEMPERATURE LIMIT}

Consider the low-temperature limit the calculation in which is the goal of this paper. By low temperatures we mean $T \ll T_{\mathrm{U}}$, when the states of the electrons involved in the scattering are significantly distorted by the interaction with the crystal lattice. The "+-" and "-+" processes require the involvement of a phonon with a wave number $q \gtrsim$ $E_{\mathrm{g}} /(\hbar c)$ (see Fig. 4). The energies of such photons are $\hbar \omega \sim \hbar q V_{\mathrm{ph}} \sim k_{\mathrm{B}} T_{\mathrm{U}}$ and exceed significantly the thermal energy for the conditions under consideration. Therefore, the "+-" and "-+" scattering may be neglected due to the exponentially small phonon mode occupation numbers $n_{\mathbf{q}, \nu}$. For the " ++ " and "--" processes at $T \ll T_{\mathrm{U}}$, the wave numbers of the phonons making the largest contribution to the scattering are small compared to $E_{\mathbf{g}} /(c \hbar)$. The integral $I_{3}^{\mathbf{g}, \nu}$ can then be written as

$$
\begin{gathered}
I_{3}^{\mathbf{g}}=\frac{\hbar^{3} C_{\perp}^{2} c^{3}}{4 C_{\|}^{4} E_{\mathbf{g}}^{3}} \sum_{\nu} \int \frac{\left(C_{\|}^{2} q_{\|}^{2}-C_{\perp}^{2} q_{\perp}^{2}\right)^{7 / 2}}{q_{\|}^{4}} \\
\times \frac{e^{-z_{\nu}}}{\left(1-e^{-z_{\nu}}\right)^{2}}\left(\mathbf{e}_{\mathbf{q}, \nu} \cdot \mathbf{g}\right)^{2} \mathrm{~d} \varphi_{\mathbf{k}} \mathrm{d}^{3} q .
\end{gathered}
$$

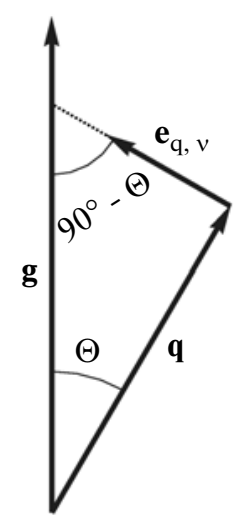

Fig. 7. Diagram showing the positions of the polarization vector and the vectors $\mathbf{q}$ and $\mathbf{g}$.

The integration is over the region $C_{\|}\left|q_{\|}\right|>$ $>C_{\perp}\left|q_{\perp}\right|$ corresponding to the " ++ " and "--" processes.

For simplicity, we use a simplified model of the phonon spectrum: $\omega_{1}=\omega_{2}=V_{\mathrm{ph}} q, \omega_{3}=\omega_{\mathrm{p}}$. The $\nu=1$ and 2 modes correspond to the transverse polarization, while the third mode corresponds to the longitudinal one. The isotropy of the phonon spectrum in this model reduces the integration over $\mathrm{d} \varphi_{\mathbf{k}}$ in (33) to the multiplication by $2 \pi$. Only the phonons of the first two modes can have frequencies $\omega \sim$ $k_{\mathrm{B}} T / \hbar$, while the contribution to the integral $I_{3}^{\mathrm{g}}$ from the third mode is exponentially small $\propto \exp \left(-T_{\mathrm{p}} / T\right)$.

Let us introduce a spherical coordinate system for the vector $\mathbf{q}$ with the polar angle $\theta$ measured from $\mathbf{e}_{\varphi}=\left[\mathbf{e}_{\|} \times \mathbf{e}_{\perp}\right]$ and the azimuthal angle $\varphi$ measured from the vector $\mathbf{e}_{\|}$(see Fig. 6). Here, $\mathbf{e}_{\|}, \mathbf{e}_{\perp}$, and $\mathbf{e}_{\varphi}$ are the unit vectors of the local Cartesian coordinate system introduced in Section 3. The cosine of the angle $\Theta$ between $\mathbf{q}$ and $\mathbf{g}$ is $\cos (\Theta)=q_{\| \mid} / q=$ $\sin (\theta) \cos (\varphi)$. Since the transverse phonons are degenerate in our case, their polarization vectors can be chosen arbitrarily in a plane perpendicular to the vector q. For simplicity, let us choose the polarization vector of the first acoustic mode to be perpendicular to the vector $\mathbf{g}$. The polarization vector of the second acoustic mode then lies in the $(\mathbf{g}, \mathbf{q})$ plane. Accordingly (see Fig. 7), $\left(\mathbf{e}_{\mathbf{q}, \nu} \cdot \mathbf{g}\right)^{2}=\sin ^{2}(\Theta) g^{2}$. Only this mode contributes to the integral $I_{3}^{\mathbf{g}, \nu}$. After the passage to the variables $q, \theta$, and $\varphi$, the latter can be taken analytically and the Coulomb logarithm can be written as

$$
\begin{aligned}
\Lambda_{\sigma}= & \frac{135 \pi^{2} \zeta(5)}{\alpha_{f}^{3}} \frac{p_{\mathrm{F}}^{2}}{2 m_{\mathrm{i}} k_{\mathrm{B}} T}\left(\frac{k_{\mathrm{B}} T}{V_{\mathrm{ph}} p_{\mathrm{F}}}\right)^{6} \\
& \times \sum_{\mathbf{g}} \mathrm{e}^{W(g)} \frac{\mathfrak{V}\left(C_{\|}\right)}{F(g)}\left(1+\frac{k_{\mathrm{TF}}^{2}}{g^{2}}\right) .
\end{aligned}
$$


Here, $\zeta(5) \approx 1.037$ is the value of the Riemann zeta function and we introduced the function

$$
\begin{gathered}
\mathfrak{V}\left(C_{\|}\right) \\
=C_{\perp}^{2}\left[\left(54 C_{\perp}^{6}+92 C_{\perp}^{4}-2 C_{\perp}^{2}\right) K\left(C_{\|}^{2}\right)\right. \\
\left.-\left(3 C_{\perp}^{6}+118 C_{\perp}^{4}-27 C_{\perp}^{2}+4\right) E\left(C_{\|}^{2}\right)\right],
\end{gathered}
$$

in which $K(x) \equiv \int_{0}^{\pi / 2}\left[1-x \sin ^{2}(t)\right]^{-1 / 2} \mathrm{~d} t$ and $E(x) \equiv \int_{0}^{\pi / 2}\left[1-x \sin ^{2}(t)\right]^{1 / 2} \mathrm{~d} t$ are complete elliptic integrals of the first and second kinds.

Note that the final expression for the Coulomb logarithm (34) is inversely proportional to the Fourier transform of the scattering potential $\phi(\mathrm{g})$ (therefore, for example, the form factor of the atomic nuclei $F(q)$ turns out to be in the denominator of Eq. (34)) rather than to $\phi^{2}(\mathrm{~g})$, just as in the approximation of free electrons (32). This is true only in the lowtemperature limit but also remains valid without using the model phonon spectrum, because the coefficient $1 / E_{\mathbf{g}}^{3}$ is taken outside the integral sign before using any assumptions about the phonon spectrum (see Eq. (33)). It can be shown that during the scattering by phonons with a small wave vector $\mathbf{q}$, the factors $F_{u v}^{\mathbf{g}}$ for the most significant (at low temperatures) " ++ " and "-_" processes turn out to be proportional to $\propto 1 / \phi(\mathrm{g})$ (see Appendix A). This cancels out with the explicit dependence of the scattering matrix elements on $\phi(\mathrm{g})$, while the remaining dependence of the Coulomb logarithm $\propto 1 / \phi(\mathrm{g})$ comes from the velocity difference $\left|\mathbf{V}_{\mathbf{k}}-\mathbf{V}_{\mathbf{k}^{\prime}}\right|$.

According to Eq. (34), the electron-phonon scattering rate due to Umklapp processes at low temperatures turns out to be proportional to $T^{5}$ rather than freezes exponentially, as was suggested by Gnedin et al. (2001). At low temperatures, the Coulomb logarithm determined by the normal electron-phonon processes is also proportional to $T^{5}$ (see Eq. (41) and Raikh and Yakovlev 1982). However, since the normal processes lead only to slow momentum diffusion over the Fermi surface, the Umklapp processes considered here also dominate at low temperatures. The results are compared in detail in Section 7.

For the subsequent investigation, we will approximate the function $\mathfrak{V}\left(C_{\|}\right)$by the expression

$$
\mathfrak{V}\left(C_{\|}\right) \approx 4\left(1-C_{\|}^{2}\right) \exp \left[12\left(C_{\|}-1\right)\right] .
$$

The accuracy of approximation (36) is illustrated in Fig. 8. It shows the exact values of the functions $\mathfrak{V}\left(C_{\|}\right), C_{\|}^{2} \mathfrak{V}\left(C_{\|}\right)$, and $\mathfrak{V}\left(C_{\|}\right)\left[20\left(1-C_{\|}^{2}\right)\right]$ (symbols) and the values corresponding to the approximation (lines). We see that all functions have a maximum in a fairly narrow region, $0.7 \lesssim C_{\|} \lesssim 1$, where

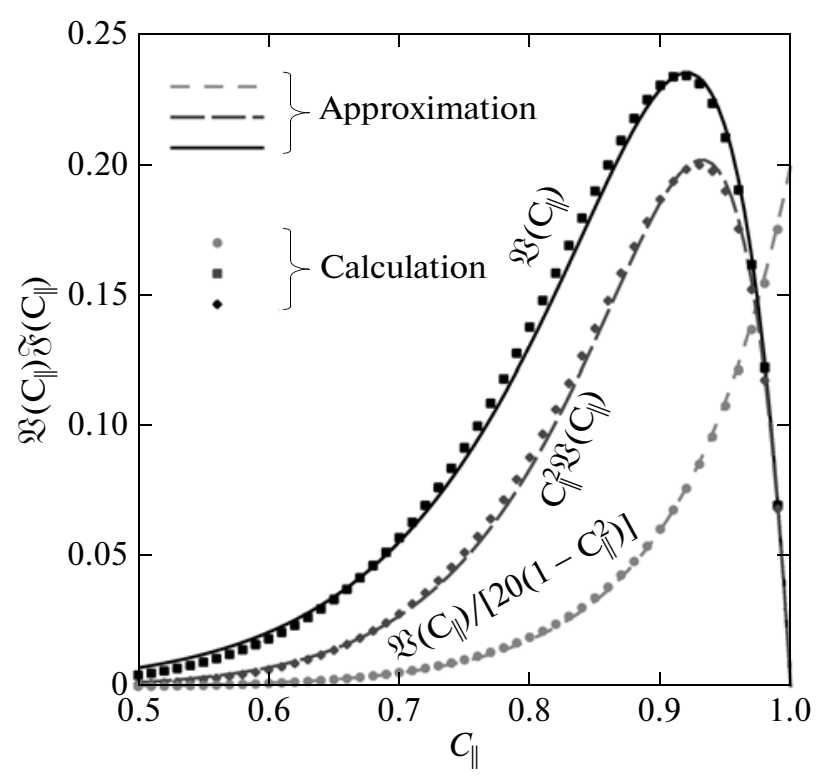

Fig. 8. Function $\mathfrak{V}\left(C_{\|}\right)$(squares) and its approximation (36) (solid line). The functions $C_{\|}^{2} \mathfrak{V}\left(C_{\|}\right)$and $\mathfrak{V}\left(C_{\|}\right)\left[20\left(1-C_{\|}^{2}\right)\right]$ (symbols) are also shown on the plot. The corresponding quantities obtained using approximation (36) are indicated by the lines.

approximation (36) allows the exact values of the corresponding functions to be well reproduced. This region corresponds to $g \gtrsim k_{\mathrm{F}}$ and makes a major contribution to the electron scattering. Since $k_{\mathrm{TF}}^{2} / g^{2} \sim$ $4 \alpha_{\mathrm{f}} / \pi \ll 1$ in it, the electron screening may be neglected. Following Chugunov and Haensel (2007), we will represent the form factor of the atomic nuclei as $F(g)=\exp \left(-w_{\text {form }} C_{\|}^{2} / 2\right)$, where the parameter $w_{\text {form }}=43 r_{\mathrm{p}}^{2} / a^{2}$ depends on the density. Here, $r_{\mathrm{p}}$ is the equivalent radius of the proton distribution in the atomic nucleus (the radius of a nucleus with a uniform proton density in which the same root-meansquare radius of the charge distribution as that in real atomic nuclei at a given mass density $\rho$ is reached). The approximation of the density dependence of $r_{\mathrm{p}}$ for the smooth composition model is given in Appendix B in the monograph by Haensel et al. (2007)). We will write the Debye-Waller factor as $W(g)=$ $w_{\text {DW }} C_{\|}^{2} / 2$. In the low-temperature limit under consideration, $w_{\text {DW }} \approx 2.4\left(A_{\text {nuc }} Z / x_{\mathrm{r}}\right)^{-1 / 2}$.

Equation (34) can be simplified by taking into account the fact that many terms enter into the sum over $\mathbf{g}$ (because $q_{\mathrm{Bz}}{ }^{3} \ll\left(2 k_{\mathrm{F}}\right)^{3}$ ) and the summation can be approximately replaced by the integration over the vectors $\mathbf{g}$ by assuming them to be distributed uniformly in $k$ space with a constant concentration $n_{\mathbf{g}}=\left(8 \pi^{3} n_{\mathrm{i}}\right)^{-1}$. The corresponding integral can be 


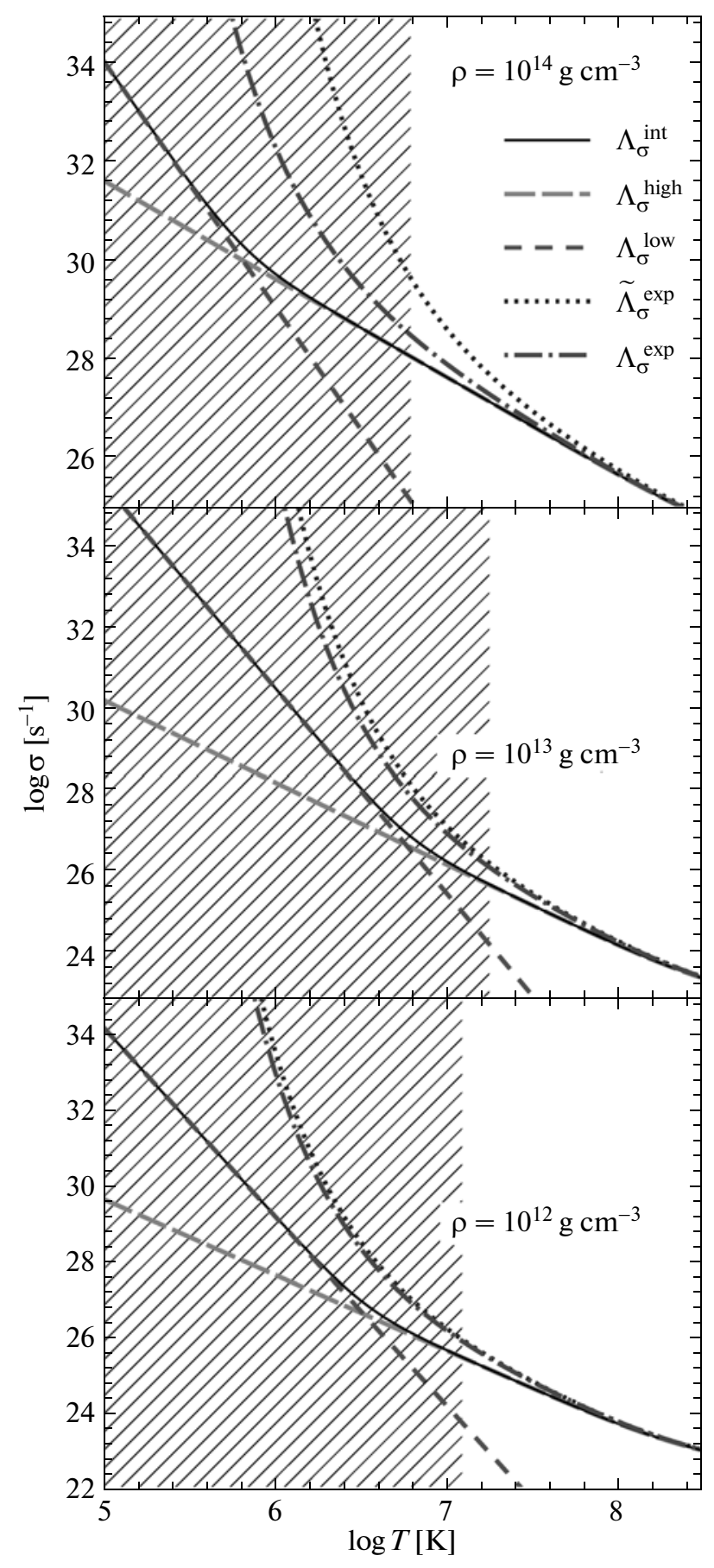

Fig. 9. Electrical conductivity $\sigma$ versus temperature in a neutron star crust with a smooth nuclear composition at densities $\rho=10^{12}, 10^{13}$, and $10^{14} \mathrm{~g} \mathrm{~cm}^{-3}$. The solid line indicates interpolation (39); the long and short dashes indicate the high- and low-temperature asymptotics $\Lambda_{\sigma}^{\text {high }}$ and $\Lambda_{\sigma}^{\text {low }}(37)$. The dash-dotted and dotted lines indicate the calculations in the exponential freezing model without and with allowance made for the influence of the form factor of atomic nuclei on the temperature $T_{\mathrm{U}}$ ( $\Lambda_{\sigma}^{\exp }$ and $\tilde{\Lambda}_{\sigma}^{\exp }$ ). The regions $T \leq T_{\mathrm{U}}$ are hatched. taken analytically (using approximation (36)), but the integration result is too cumbersome. Nevertheless, it is well approximated by the expression

$$
\Lambda_{\sigma}=5 \alpha_{\mathrm{f}}^{2} \frac{Z^{2 / 3}}{C^{6}} \frac{p_{\mathrm{F}}^{2}}{m_{\mathrm{i}} k_{\mathrm{B}} T_{\mathrm{p}}}\left(\frac{T}{\widetilde{T}_{\mathrm{U}}}\right)^{5} \mathrm{e}^{0.4 w},
$$

where $w=w_{\text {form }}+w_{\mathrm{DW}}$ and $C=V_{\mathrm{ph}} q_{\mathrm{BZ}} / \omega_{\mathrm{p}}$. In the low-temperature limit under consideration, the influence of the Debye-Waller factor is negligible $\left(w_{\text {DW }} \lesssim\right.$ 0.15 ), whereas the form factor of the atomic nuclei near the boundary of a crust with a neutron star core corresponds to $w_{\text {form }} \lesssim 8$, which increases significantly the Coulomb logarithm.

As the effective phonon velocity $V_{\text {ph }}$, we will take the phase velocity $\omega_{\nu} / q$ for $q \rightarrow 0$ averaged over the directions of the wave vector $\mathbf{q}$ and the polarizations of the acoustic phonons $\nu=1,2$ :

$$
V_{\mathrm{ph}}=\lim _{q \rightarrow 0}\left[\frac{1}{2} \sum_{\nu} \int \frac{\mathrm{d} \Omega_{\mathbf{q}}}{4 \pi}\left(\frac{\omega_{\nu}}{q}\right)^{-5}\right]^{-1 / 5} .
$$

The corresponding constant $C \approx 0.36$ (Chugunov and Haensel 2007). This quantity and, hence, the electrical conductivity is virtually independent of the type of crystal lattice of atomic nuclei (bcc or fcc). Note that this conclusion is valid owing to the simplifying assumptions made in this section. A more accurate calculation with allowance made for the true phonon spectrum and with direct summation over the reciprocal lattice vectors can reveal a dependence on the type of lattice, but it is unlikely that this dependence will be significant for applications.

\section{ANALYSIS OF RESULTS}

Figure 9 shows the temperature dependence of the electrical conductivity for matter with a smooth equilibrium nuclear composition at $\rho=10^{12}, 10^{13}$, and $10^{14} \mathrm{~g} \mathrm{~cm}^{-3}$. The regions $T \leq T_{\mathrm{U}} \approx 1.25 \times 10^{7}$, $1.8 \times 10^{7}$, and $6.2 \times 10^{6} \mathrm{~K}$, respectively, are shaded. Outside these regions, the electrical conductivity may be considered in the approximation of almost free electrons. The currently most accurate calculation of the Coulomb logarithm $\Lambda_{\sigma}^{\text {high }}$ for matter in the outer neutron star crust in this approximation was made by Potekhin et al. (1999); they also proposed an approximation of the results of their calculation. The Coulomb logarithms in the inner neutron star crust were calculated and approximated in Appendix A to the succeeding paper by Gnedin et al. (2001). These results were realized in the form of a publicly accessible code in Fortran ${ }^{2}$. In Fig. 9, they are indicated

\footnotetext{
${ }^{2}$ http://www.ioffe.ru/astro/conduct/
} 
by the line with long dashes. At temperatures $T \ll$ $T_{\mathrm{U}}$, the Coulomb logarithm was calculated here (see Eq. (37)). We will denote the Coulomb logarithm formally calculated from this formula at all temperatures by $\Lambda_{\sigma}^{\text {low }}$. The corresponding electrical conductivity is indicated in Fig. 9 by the short dashes. In the intermediate range of temperatures, the Coulomb logarithm should be calculated numerically in accordance with Eqs. (27) and (30) and by taking into account the exact spectrum and polarization of phonon modes (see, e.g., Baiko et al. 2001; Baiko 2002). However, the Coulomb logarithm at intermediate temperatures can be estimated on the basis of a simple interpolation:

$$
\Lambda_{\sigma}^{\text {int }} \approx\left[\frac{1}{\Lambda_{\sigma}^{\text {high }}}+\frac{1}{\Lambda_{\sigma}^{\text {low }}}\right]^{-1} .
$$

This interpolation reproduces the limits of high $(T \gg$ $\left.T_{\mathrm{U}}\right)$ and low $\left(T \ll T_{\mathrm{U}}\right)$ temperatures and describes the smooth dependence of the Coulomb logarithm on parameters indicated in Fig. 9 by the solid line in the intermediate range. We see that the asymptotics $\Lambda_{\sigma}^{\text {high }}$ and $\Lambda_{\sigma}^{\text {low }}$ (the long and short dashes, respectively) limit the electrical conductivity quite reliably at intermediate temperatures. It is hoped that the interpolation is valid at all temperatures, at least in order of magnitude. Note that the transition from $\Lambda_{\sigma}^{\text {high }}$ to $\Lambda_{\sigma}^{\text {low }}$ occurs at temperatures well below $T_{\mathrm{U}}$. This is because the reciprocal lattice vectors $g \sim 1.8 k_{\mathrm{F}}$ (Fig. 8) to which smaller gap widths than $E_{\text {gap }}$ used in estimate (16) correspond make a major contribution to the scattering at low temperatures.

The dash-dotted line in Fig. 9 indicates the electrical conductivity calculated within the model of exponential freezing of the scattering processes proposed by Gnedin et al. (2001) for the description of low temperatures $T \lesssim T_{\mathrm{U}}$. The corresponding Coulomb logarithm can be written as

$$
\begin{gathered}
\Lambda_{\sigma}^{\exp }=\Lambda_{\sigma}^{\text {high }} \exp \left(-\frac{T_{\mathrm{U}}}{T}\right) \\
+\Lambda_{\sigma}^{\text {norm }}\left[1-\exp \left(-\frac{T_{\mathrm{U}}}{T}\right)\right],
\end{gathered}
$$

where

$$
\Lambda_{\sigma}^{\text {norm }}=\frac{360 \zeta(5)}{Z x_{\mathrm{r}}} \frac{k_{\mathrm{B}} T}{m_{\mathrm{e}} c^{2}}\left(\frac{T}{T_{\mathrm{p}}}\right)^{4}
$$

is the Coulomb logarithm determined by the normal electron-phonon scattering processes (Raikh and Yakovlev 1982). In reality, the numerical value of $\Lambda_{\sigma}^{\text {norm }}$ turns out to be very low:

$$
\frac{\Lambda_{\sigma}^{\text {norm }}}{\Lambda_{\sigma}^{\text {low }}} \approx 2.4 \times 10^{-11} \frac{Z}{30}\left(\frac{C}{0.36}\right)^{6} \mathrm{e}^{-0.4 w}
$$

and does not affect the result.

Note that Gnedin et al. (2001) disregarded the influence of the form factor of atomic nuclei on the temperature $T_{\mathrm{U}}$ and we should set $T_{\mathrm{U}}=\widetilde{T}_{\mathrm{U}}$ in our calculation using Eq. (40) for strict correspondence to their results. The electrical conductivity corresponding to this Coulomb logarithm $\widetilde{\Lambda}_{\sigma}^{\exp }$ is indicated by the dotted line. According to Fig. 9, using the model of exponential reduction of the scattering processes leads to an overestimation of the electrical conductivity at low temperatures by several orders of magnitude, especially when using the Coulomb logarithm $\tilde{\Lambda}_{\sigma}^{\exp }$.

The Coulomb logarithm $\Lambda_{\sigma}^{\text {norm }}$ is determined by the normal processes occurring at all temperatures on the entire Fermi surface rather than concentrating near the boundaries of the Brillouin zones. This scattering mechanism was not considered here. In the approximation of uncorrelated scattering, the corresponding electron relaxation rate should be added to the calculated one. This is equivalent to the addition of the corresponding Coulomb logarithms. However, the numerical value of $\Lambda_{\sigma}^{\text {norm }}$ turns out to be lower than $\Lambda_{\sigma}^{\text {low }}$ by ten orders of magnitude (see Eq. (42)), because the distribution function is relaxed through slow momentum diffusion due to the large number of small-angle scatterings. Therefore, the normal processes, just as at high temperatures (see, e.g., Potekhin et al. 1999), have no effect on the electrical conductivity.

Figure 10 presents the density dependence of the electrical conductivity for matter with a smooth equilibrium nuclear composition. As an example, we chose the temperatures $T=3 \times 10^{7}, 10^{7}$, and $3 \times$ $10^{6} \mathrm{~K}$. Just as in Fig. 9, the long and short dashes correspond to the high- and low-temperature asymptotics of the Coulomb logarithm, $\Lambda_{\sigma}^{\text {high }}$ and $\Lambda_{\sigma}^{\text {low }}$, while the solid line indicates interpolation (39). The dash-dotted line indicates the electrical conductivity in the model of exponential freezing of Umklapp processes $\Lambda_{\sigma}^{\exp }$ (Gnedin et al. 2001). At $T=3 \times$ $10^{6} \mathrm{~K}$ and $\rho \sim 10^{13} \mathrm{~g} \mathrm{~cm}^{-3}$, this model leads to an overestimation of the electrical conductivity by more than two orders of magnitude. However, the electrical conductivities in the exponential model (40) and interpolation (39) differ by only an order of magnitude already at $T \sim 10^{7} \mathrm{~K}$ and by no more than several times at $T \sim 3 \times 10^{7} \mathrm{~K}$. Finally, at $T \sim 10^{8} \mathrm{~K}$ 


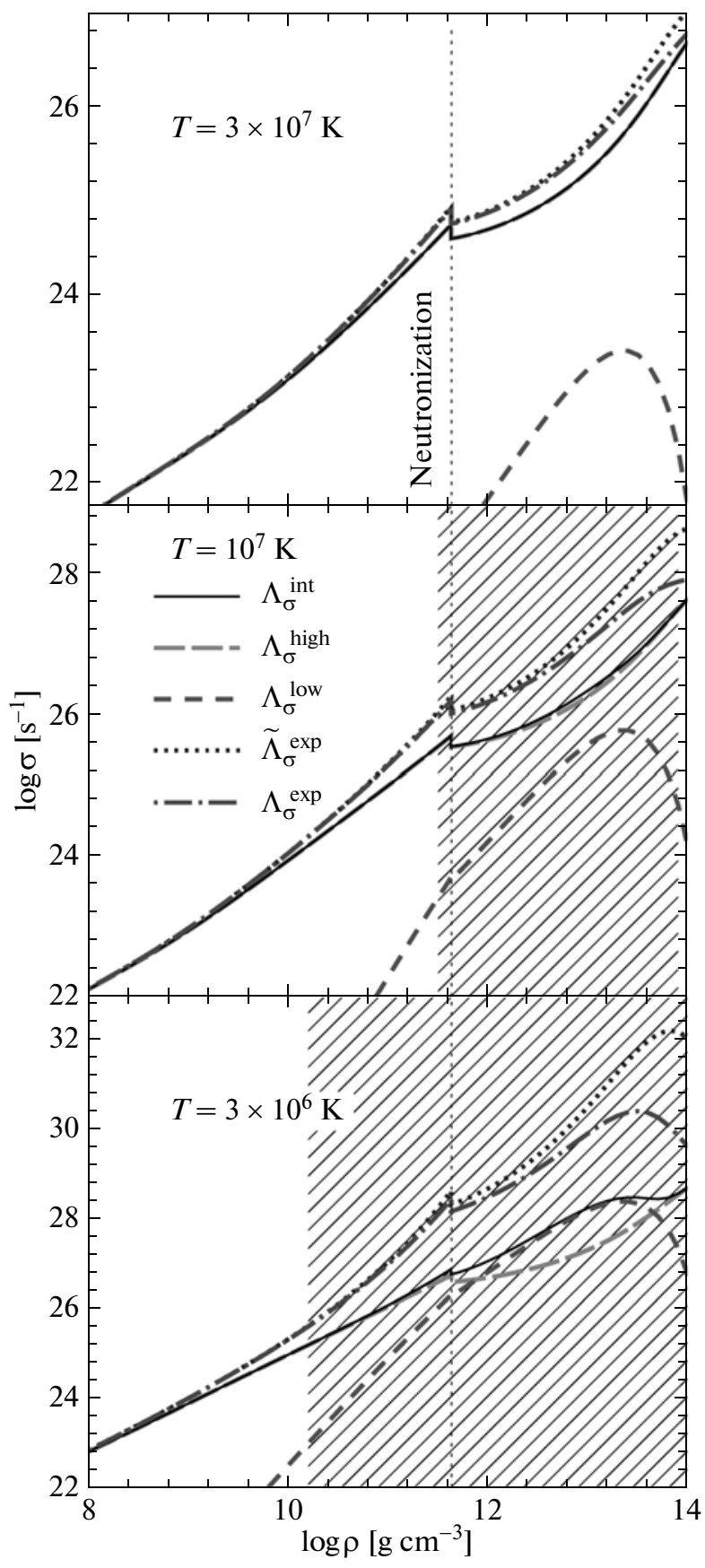

Fig. 10. Electrical conductivity $\sigma$ versus density $\rho$ in a neutron star crust with a smooth equilibrium nuclear composition at temperatures $T=3 \times 10^{7}, 10^{7}$, and $3 \times$ $10^{6} \mathrm{~K}$. The solid line indicates interpolation (39); the short dashes, long dashes, the dotted and dash-dotted lines indicate the electrical conductivities corresponding to the Coulomb logarithms $\Lambda_{\sigma}^{\text {high }}, \Lambda_{\sigma}^{\text {low }}$ and $\tilde{\Lambda}_{\sigma}^{\exp }, \Lambda_{\sigma}^{\exp }$. The regions of densities at which $T<T_{\mathrm{U}}$ (for $3 \times 10^{7} \mathrm{~K}$, there is no such region) are hatched. The vertical dotted line indicates the neutronization density $\rho_{\mathrm{d}}=4.3 \times$ $10^{11} \mathrm{~g} \mathrm{~cm}^{-3}$. (not shown in Fig. 10) exceeding considerably the maximum value of $T_{\mathrm{U}} \sim 1.8 \times 10^{7} \mathrm{~K}$ (see Fig. 1), the electrical conductivity is determined by the hightemperature asymptotics $\Lambda_{\sigma}^{\text {high }}$ derived in the approximation of free electrons (Potekhin et al. 1999; Gnedin et al. 2001). In this case, the model of exponential reduction of Umklapp processes (40) and interpolation (39) yield virtually the same result.

The dotted line corresponding to the Coulomb logarithm $\widetilde{\Lambda}_{\sigma}^{\exp }$ demonstrates an even greater overestimation of the electrical conductivity relative to the calculation based on interpolation (39).

In Fig. 10, the regions corresponding to $T<T_{\mathrm{U}}$ are hatched. As has already been discussed above, the widely used approximation of free electrons outside these regions is justified and interpolation (39) proposed here coincides with the result obtained in this approximation by Potekhin et al. (1999) and Gnedin et al. (2001). However, already at $T=T_{\mathrm{U}}$, using the model of exponential freezing of scattering leads to a noticeable (by a factor of 2.7 ) overestimation of the electrical conductivity.

The thin vertical dotted line in Fig. 10 indicates the neutronization density $\rho_{\mathrm{d}}=4.3 \times 10^{11} \mathrm{~g} \mathrm{~cm}^{-3}$. When passing through it, the composition of the matter changes abruptly (free neutrons appear), which is accompanied by a jump in electrical conductivity and other transport coefficients (see, e.g., Gnedin et al. 2001).

\section{CONCLUSIONS}

Using the variational method, we calculated the electrical conductivity of the neutron star crust in the low-temperature limit, $T \ll T_{\mathrm{U}}$ (Section 6 ). In our calculation, we took into account the influence of the static lattice of atomic nuclei on the electron wave functions (Section 3). We showed that the electrical conductivity does not depend on the type of crystal lattice. We proposed the interpolation formula (39) that allows the electrical conductivity to be calculated at any temperatures. We demonstrated that the previously existed model of exponential reduction of the scattering rate (Gnedin et al. 2001) could overestimate the electrical conductivity by several orders of magnitude (Section 7); the causes of the imperfection of this model are pointed out (Section 4). 
The results obtained are important for investigating the magnetic field decay in neutron stars with internal temperatures $T \lesssim 10^{7} \mathrm{~K}$ that can manifest themselves as pulsars. In addition, the crustal electrical conductivity is important for constructing models for the spread of the currents flowing along the pulsar tube over the neutron star crust. Analysis of the constraints on the sizes of the mountains maintained by the crustal conductivity in accreting millisecond pulsars (Vegelius and Melatos 2010) can become another application of the electrical conductivity calculation presented here.

In future, we are planning to generalize our results to other transport coefficients (thermal conductivity and shear viscosity) that have been previously calculated in the approximation of free electrons (Potekhin et al. 1999; Chugunov and Yakovlev 2005) or using the model of exponential freezing of the electronphonon scattering (Gnedin et al. 2001). Just as for the electrical conductivity, this model can lead to an overestimation of the transport coefficients by several orders of magnitude. In addition, we are planning to consider the influence of the electron-phonon Umklapp processes that have not been considered previously in investigating the electron-electron collisions in a neutron star crust (Shternin and Yakovlev 2006; Shternin 2008), but can be important at low temperatures (see, e.g., Landau and Pomeranchuk 1937).

\section{APPENDIX A}

\section{THE MATRIX ELEMENT \\ AND THE TRANSITION PROBABILITY}

We use the periodic zone scheme and consider the scattering by a phonon with a small wave vector q. In this case, electrons with close quasi-momenta are involved in the scattering and the relation $\mathbf{p}+$ $\hbar \mathbf{q}=\mathbf{p}^{\prime}$ is valid (in the case of phonon absorption). The electron-phonon scattering probability can be represented as

$$
\begin{gathered}
P_{\mathbf{p}, \mathbf{q}, \nu}^{\mathbf{p}^{\prime}}=\frac{2 \pi}{\hbar}\left|M_{\mathbf{q}}^{(i)\left(i^{\prime}\right)}\left(\mathbf{p}, \mathbf{p}^{\prime}\right)\right|^{2} \\
\times f_{\mathbf{p}}\left(1-f_{\mathbf{p}^{\prime}}\right) \delta\left(E_{\mathbf{p}}+\hbar \omega_{\mathbf{q}, \nu}-E_{\mathbf{p}^{\prime}}\right),
\end{gathered}
$$

where the electron state indices before and after scattering $(i)$ and $\left(i^{\prime}\right)$ in $P_{\mathbf{p}, \mathbf{q}, \nu}^{\mathbf{p}^{\prime}}$ are omitted for brevity and the transition matrix element is (Ziman 1960)

$$
\begin{gathered}
M_{\mathbf{q}}^{(i)\left(i^{\prime}\right)}\left(\mathbf{p}, \mathbf{p}^{\prime}\right)=\frac{n_{\mathrm{i}} n_{\mathbf{q}, \nu}^{1 / 2}}{2 \epsilon_{\mathbf{k}}}\left(\frac{\hbar}{m_{\mathrm{i}} n_{\mathrm{i}} V \omega_{\mathbf{q}, \nu}}\right)^{1 / 2} \\
\times J_{\mathbf{q}, \nu}^{(i)\left(i^{\prime}\right)}\left(\mathbf{p}, \mathbf{p}^{\prime}\right)(2 \pi)^{4} \delta_{\mathbf{k}-\mathbf{k}^{\prime}+\mathbf{q}}
\end{gathered}
$$

Here, $V$ is the normalization volume, $\omega_{\mathbf{q}, \nu}$ and $\mathbf{e}_{\mathbf{q}, \nu}$ are the phonon frequency and polarization vector, and

$$
J_{\mathbf{q}, \nu}^{(i)\left(i^{\prime}\right)}\left(\mathbf{p}, \mathbf{p}^{\prime}\right)=-\mathbf{e}_{\mathbf{q}, \nu}\left\langle\Psi_{\sigma, \mathbf{p}}^{(i)}\left|\nabla U_{a}\right| \Psi_{\sigma^{\prime}, \mathbf{p}^{\prime}}^{\left(i^{\prime}\right)}\right\rangle .
$$

Here, $\sigma$ and $\sigma^{\prime}$ are the helicities before and after scattering. After the substitution of the electron wave functions (14) in the initial and final states, we obtain

$$
\begin{gathered}
\left|J_{\mathbf{q}, \nu}^{++}\left(\mathbf{p}, \mathbf{p}^{\prime}\right)\right|^{2} \approx\left|J_{\mathbf{q}, \nu}^{--}\left(\mathbf{p}, \mathbf{p}^{\prime}\right)\right|^{2} \\
\approx 4 \epsilon^{2}\left(\mathbf{e}_{\mathbf{q}, \nu} \cdot \mathbf{g}\right)^{2} U_{g}^{2} C_{\perp}^{2}\left(v_{\mathbf{k}} u_{\mathbf{k}^{\prime}}-u_{\mathbf{k}} v_{\mathbf{k}^{\prime}}\right)^{2} \delta_{\sigma, \sigma^{\prime}} \\
\left|J_{\mathbf{q}, \nu}^{+-}\left(\mathbf{p}, \mathbf{p}^{\prime}\right)\right|^{2} \approx\left|J_{\mathbf{q}, \nu}^{-+}\left(\mathbf{p}, \mathbf{p}^{\prime}\right)\right|^{2} \\
\approx 4 \epsilon^{2}\left(\mathbf{e}_{\mathbf{q}, \nu} \cdot \mathbf{g}\right)^{2} U_{g}^{2} C_{\perp}^{2}\left(v_{\mathbf{k}} v_{\mathbf{k}^{\prime}}+u_{\mathbf{k}} u_{\mathbf{k}^{\prime}}\right)^{2} \delta_{\sigma, \sigma^{\prime}}
\end{gathered}
$$

Thus, the helicity is conserved during scattering. In Eqs. (A4), we neglect the terms containing $U_{\mathbf{q}}\left(\mathbf{e}_{\mathbf{q}, \nu} \cdot \mathbf{q}\right)$. For low-frequency transverse phonons, they are small due to the transverse polarization $\left(\mathbf{e}_{\mathbf{q}, \nu} \cdot \mathbf{q}\right) \propto q^{2} / q_{\mathrm{BZ}}$ (see, e.g., Baiko 2000). Indeed, for $T \lesssim T_{\mathrm{U}}$, the typical phonon wave numbers $q \ll$ $k_{\mathrm{TF}}$. Therefore, owing to the electron screening, $U_{\mathbf{q}} \propto 1 / k_{\mathrm{TF}}^{2}$ and $U_{\mathbf{q}}\left(\mathbf{e}_{\mathbf{q}, \nu} \cdot \mathbf{q}\right) \propto q^{2} /\left(k_{\mathrm{TF}}^{2} q_{\mathrm{BZ}}\right)$, which is much smaller than the main term $U_{\mathbf{g}}\left(\mathbf{e}_{\mathbf{q}, \nu} \cdot \mathbf{g}\right) \propto$ $g^{-1} \sim q_{\mathrm{BZ}}{ }^{-1}$ we included. For longitudinal phonons, $\left(\mathbf{e}_{\mathbf{q}, \nu} \cdot \mathbf{q}\right) \approx q$ and the contributions to $\left|J_{\mathbf{q}}^{(i)\left(i^{\prime}\right)}\left(\mathbf{p}, \mathbf{p}^{\prime}\right)\right|^{2}$ from the terms proportional to $U_{\mathbf{q}}^{2} q^{2}$ and $q U_{\mathbf{q}} U_{\mathbf{g}}\left(\mathbf{e}_{\mathbf{q}, \nu} \cdot \mathbf{g}\right)$ should be considered separately. The first of them $U_{\mathbf{q}}^{2} q^{2}$ describes the normal scattering processes that can occur on the entire Fermi surface (and not only in the regions of its intersection with the boundaries of the Brillouin zones). Their contribution to the scattering at low temperatures was calculated by Raikh and Yakovlev (1982) and, as was shown in Section 7 , is negligible. It can be shown that the cross-term $\propto q U_{\mathbf{q}} U_{\mathbf{g}}\left(\mathbf{e}_{\mathbf{q}, \nu} \cdot \mathbf{g}\right)$ at $T \lesssim T_{\mathrm{U}}$ also makes a negligible contribution, because the phase velocity of the longitudinal phonons $\sim \omega_{\mathrm{p}} / k_{\mathrm{TF}}$ is much higher than that of the transverse ones.

Since the matrix element (A4) contains the reciprocal lattice vector, we will call the corresponding processes "Umklapp processes". Strictly speaking, this name is not always precise- the reciprocal lattice vector can be absent in the quasi-momentum conservation law written in the extended Brillouin zone scheme. However, as was shown in Section 5, the Coulomb logarithm calculated using the matrix elements (A4) in the high-temperature limit reproduces the expression derived by Raikh and Yakovlev (1982) for the Umklapp processes in the approximation of free electrons. 


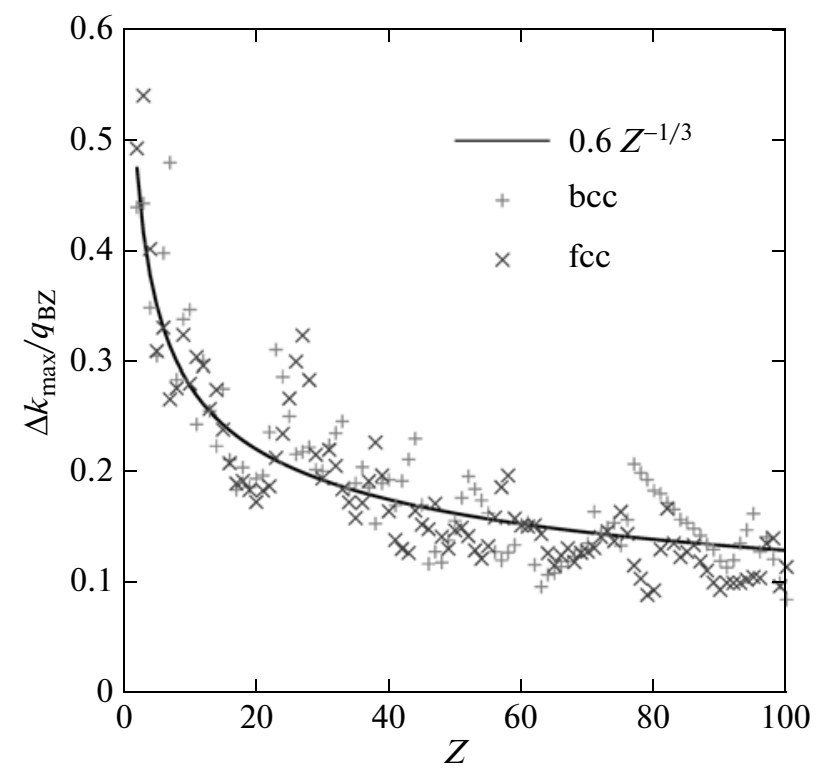

Fig. 11. Maximum distances from the point on the Fermi surface to the nearest boundary of the Brillouin zone $\Delta k_{\max }$ versus change $Z$ of atomic nuclei. The symbols + and $\times$ represent the exact calculation for bec and fec lattices; the line indicates approximation (B1).

For brevity, we will denote the corresponding functions of $u_{\mathbf{k}}, u_{\mathbf{k}^{\prime}}, v_{\mathbf{k}}, v_{\mathbf{k}^{\prime}}$ in Eqs. (A4) by $F_{u v}^{\mathbf{g}}$. For example, for the "++" processes, $\left[(j)=\left(j^{\prime}\right)=\right.$ "+"] $F_{u v}^{\mathbf{g}}=v_{\mathbf{k}} u_{\mathbf{k}^{\prime}}-u_{\mathbf{k}} v_{\mathbf{k}^{\prime}}$. The transition probability can ultimately be written as

$$
\begin{gathered}
P_{\mathbf{p}, \mathbf{q}, \nu}^{\mathbf{p}^{\prime}}=(2 \pi)^{4} \frac{n_{\mathrm{i}} n_{\mathbf{q}, \nu}}{2 m_{\mathrm{i}} \omega_{\mathbf{q}, \nu}}\left(\mathbf{e}_{\mathbf{q}, \nu} \cdot \mathbf{g}\right)^{2} \\
\times\left(F_{u v}^{\mathbf{g}}\right)^{2} U_{g}^{2} f_{\mathbf{p}}\left(1-f_{\mathbf{p}^{\prime}}\right) \delta\left(E_{\mathbf{p}}+\hbar \omega_{\mathbf{q}, \nu}-E_{\mathbf{p}^{\prime}}\right) \\
\times \delta\left(\mathbf{k}^{\prime}-\mathbf{k}-\mathbf{q}\right) \delta_{\sigma, \sigma^{\prime}} .
\end{gathered}
$$

APPENDIX B

\section{ON THE CHOICE OF A TRIAL FUNCTION $\Phi_{\mathbf{k}}$}

The electron-phonon Umklapp processes considered here at low temperatures are inefficient for electrons with momenta far from the boundaries of the Brillouin zones (in the white parts of the Fermi surface in Figs. 3 and 12). Such electrons initially must bring their momentum closer to the region of efficient electron-phonon scattering (localized near the boundaries of the Brillouin zones) through a series of small-angle scatterings on the entire Fermi surface. Only then can they be subjected to the more powerful electron-phonon Umklapp processes that changes significantly the electron velocity and quasimomentum in the extended zone scheme. Both the electrons themselves and the electron-phonon normal processes can be small-angle electron scatterers in the neutron star crust. However, these processes turn out to be less efficient. Therefore, below we will consider only the electron-electron scattering.

As a result of the two-step scattering mechanism, the deviation of the electron distribution function from the equilibrium one will increase with distance from the boundaries of the Brillouin zones. In the variational formalism, this manifests itself in the fact that the value of the trial function $\Phi_{\mathbf{k}}$ at the points farthest from the boundaries can be much higher than that near the boundaries. This, in turn, will lead to an increase in the integral $\mathbf{I}_{1}$ in Eq. (17) and, hence, to an increase in the electrical conductivity. Moreover, if we choose a trial function that is equal to zero at distances from the boundaries of the Brillouin zones smaller than some value $k_{\mathrm{m}}$, then only the phonons with a wave number $q>k_{\mathrm{m}}$ can be involved in the relaxation of the corresponding distribution function through the electron-phonon Umklapp processes. Accordingly, the integral $I_{2}$ in the low-temperature limit, $T \ll V_{\mathrm{ph}} k_{\mathrm{m}} / k_{\mathrm{B}}$, will become exponentially small $\propto \exp \left(-V_{\mathrm{ph}} k_{\mathrm{m}} /\left(k_{\mathrm{B}} T\right)\right)$, while the electrical conductivity will become exponentially large. However, if we calculate the integral $I_{2}$ describing the generation of entropy by taking into account the electron-electron scattering, then its value, on the contrary, will be large (while the electrical conductivity will be small) due to the rapid change in the trial function $\Phi_{\mathrm{k}}$. The true electrical conductivity corresponds to the maximum of functional (17) when all scattering (entropy generation) mechanisms are taken into account. This maximum is reached for a trial function that, on the one hand, is smooth enough for the generation of entropy in electronelectron collisions to be not too large and, on the other hand, minimizes the generation of entropy during the electron-phonon scattering considered here. Such a situation can take place when considering the electrical conductivity in semiconductors, where electronelectron collisions determine the shape of the distribution function but do not impede the electrical conductivity determined by the slower scattering by impurities or phonons (see, e.g., Gantmakher and Levinson 1984). In addition, a similar phenomenon also emerges when considering the thermal conductivity of a lattice attributable to the scattering by impurities (see Section 6, Chapter 8, in the book by Ziman (1960)). 


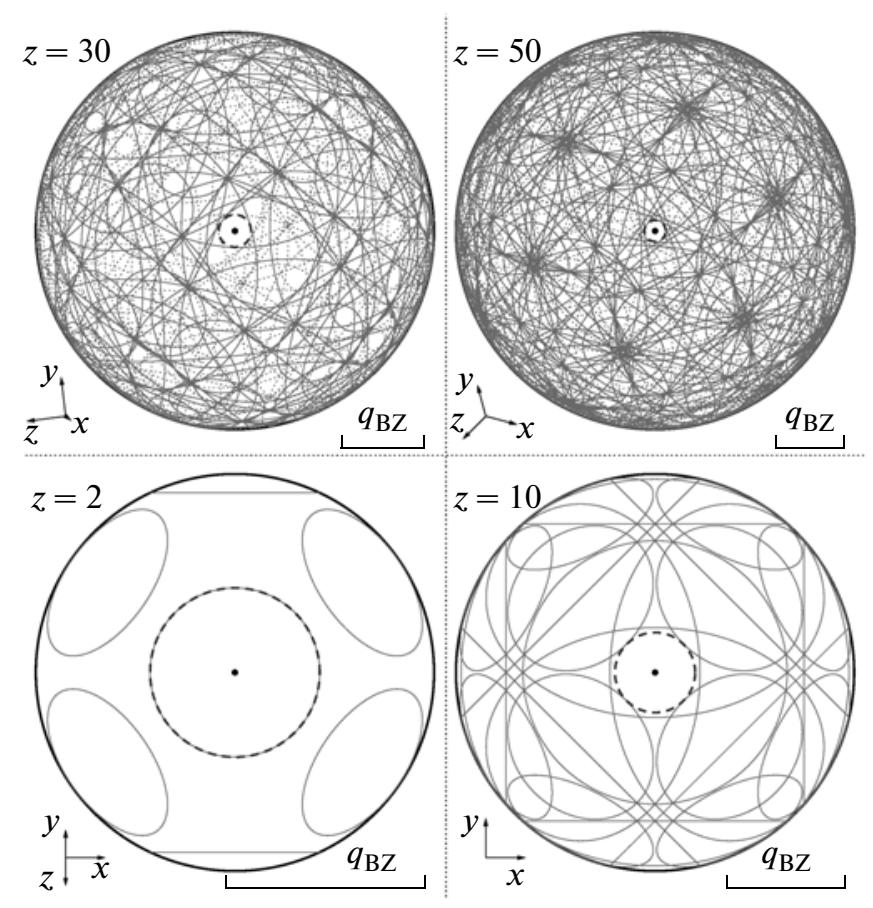

Fig. 12. Lines of intersection between the boundaries of the Brillouin zones and the Fermi sphere oriented in such a way that the wave vector farthest from the boundaries of the Brillouin zones is perpendicular to the plane of the figure. The solid lines correspond to the part of the Fermi surface facing us; the dotted lines run over its far side. As an example, we took the charges $Z=2,10,30$, and 50 . For $Z=2$ and 10 , the dotted lines coincide with the solid ones, because the crystal symmetry plane coincides with the plane of the figure. The coordinate axes corresponding to the edges of a bcc lattice are shown in the lower left corner of each figure. The thickness of the lines on the scale of the figure is equal to twice the width of the region of the greatest distortion of the electron wave functions $2 \Delta k_{\text {mix }}$. The quantity $q_{\mathrm{Bz}}$ is shown in the lower left corner of each figure; the point indicates the center of the Fermi sphere. The dashed circumference at the center has the radius $\Delta k_{\max }$.

When choosing a smooth trial function $\Phi_{\mathbf{k}}$ in Section 4 , we implicitly assume that for all points on the Fermi surface, the nearest boundary of the Brillouin zone is close enough for the electron-electron scattering to prevent a significant change in the trial function on this scale. In other words, the electronelectron scattering must have time to smooth the distribution function. This assumption is justified in a wide range of parameters, because the Fermi surface is often intersected by the numerous boundaries of high Brillouin zones owing to the large number of electrons $Z \sim 30$ per crystal lattice site (atomic nucleus) in the neutron star crust. Let us show this more rigorously.

Since the electron-electron scattering is smallangle one, the time it takes for the electron quasimomentum to change by $\Delta k$ can be estimated as $\tau_{\Delta k} \sim \tau_{\mathrm{e}}^{\text {diff }}\left[\Delta k /\left(\pi k_{\mathrm{F}}\right)\right]^{2}$. Here, $\tau_{\mathrm{e}}^{\text {diff }}$ is the time it takes for the electron momentum to rotate through $\pi$ under the action of electron-electron collisions. As the latter, we will take the electron momentum relaxation time that determines the shear viscosity. Shternin (2008) calculated this quantity by taking into account the Landau damping of transverse plasmons. If the diffusion time $\tau_{\Delta k}$ is less than the momentum relaxation time $\tau$, then our calculation with a smooth trial function $\Phi_{\mathbf{k}}$ will be justified, because electron-electron collisions for trial functions differing significantly near the boundaries of the Brillouin zones and at the points farthest from them will lead to an increase in the integral $I_{2}$ and, hence, to a poorer variational estimate of the electrical conductivity.

To obtain an upper limit for $\tau_{\Delta k}$, let us associate the distance to the nearest boundary of the Brillouin zone with each point on the Fermi sphere. We will designate the maximum distance as $\Delta k_{\max }$. For atomic nuclei with charges $Z=2-100$, the distance $\Delta k_{\max }$ was determined by the Monte Carlo method for bcc and fcc lattices and was approximated by the expression

$$
\Delta k_{\max } \sim 0.6 q_{\mathrm{BZ}} Z^{-1 / 3} .
$$

The approximation accuracy is illustrated in Fig. 11. Despite the differences between the exact calculation and the approximation by up to a factor of 1.5 , we will 


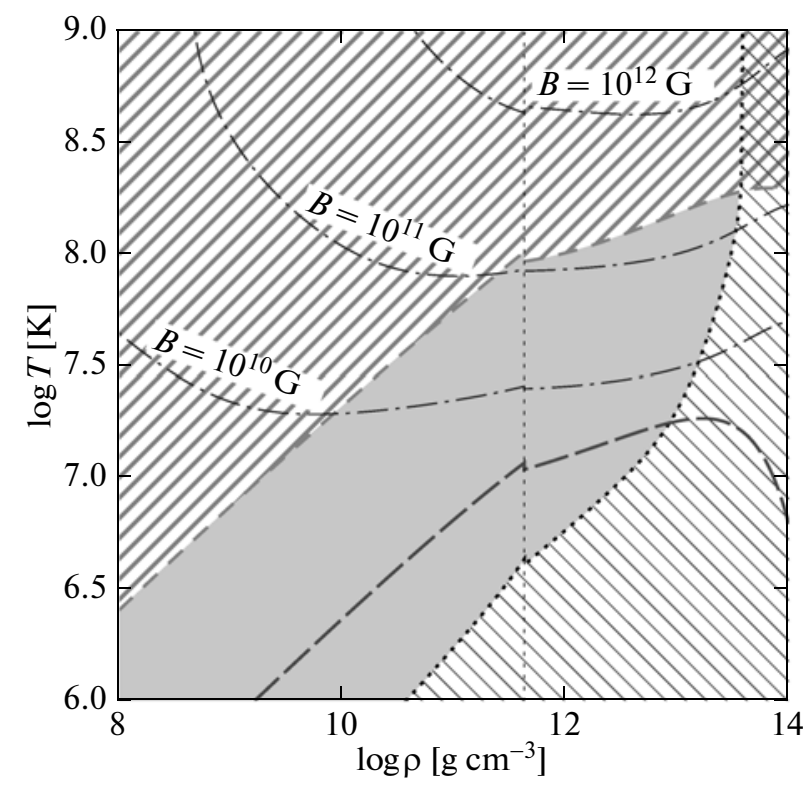

Fig. 13. Temperatures $T_{\text {ee }}$ (dotted line) and $T_{\mathrm{ph}}$ (short dashed line) versus density for a neutron star crust with a smooth equilibrium nuclear composition. In the region $T>T_{\mathrm{ph}}$ hatched by thick lines, the electronphonon scattering is fairly efficient on the entire Fermi surface. The thin hatching corresponds to the region $T<T_{\mathrm{ph}}$, where the electron-electron scattering has time to smooth the electron distribution function. In the gray region, the electron-electron scattering rate can limit the electron scattering in some parts of the Fermi surface. The long dashes indicate the temperature $T_{\mathrm{U}}$. The dashdotted lines indicate the temperatures $T_{\mathrm{B}}$ corresponding to magnetic fields $B=10^{10}, 10^{11}$, and $10^{12} \mathrm{G}$. The vertical dotted line indicates the neutronization density.

restrict ourselves to using the simple formula (B1) for the subsequent estimations. Note that the distance to the nearest boundary of the Brillouin zone is typically much smaller than $\Delta k_{\max }$. This is shown in Fig. 12, which is analogous to Fig. 3 but is oriented so that the wave vector farthest from the boundaries of the Brillouin zones is perpendicular to the plane of the figure. The dashed circumference of radius $\Delta k_{\max }$ indicates the region where there are no boundaries of the Brillouin zones. We see that many lines corresponding to the boundaries of the Brillouin zone will fall within the circumference of this radius at most other points on the Fermi surface, i.e., most other points on the Fermi surface are much closer to the boundaries of the Brillouin zones. Therefore, on the bulk of the Fermi surface, the diffusion time to such a boundary $\tau_{\Delta k}$ will be much smaller than our estimate under the assumption of $\Delta k=\Delta k_{\max }$. Consequently, the deviation of the electron distribution function from the equilibrium one (and, hence, the best trial function $\Phi_{\mathbf{k}}$ ) will be a smoother function.
Next, we will calculate the time $\tau$ using the interpolation of the Coulomb logarithm (39). If the condition $\tau_{\Delta k}=\tau$ is met at some temperature $T_{\text {ee }}$, then the relation $\tau_{\Delta k}<\tau$ will be valid at temperatures $T<T_{\text {ee }}$ due to the sharper temperature dependence of the time $\tau\left(\tau_{\Delta k} \propto T^{5 / 3}\right)$. Therefore, in the region $T<T_{\text {ee }}$ indicated by the thin hatching in Fig. 13, using a smooth trial function $\Phi_{\mathbf{k}}$ is justified.

At fairly high temperatures (the thick hatching in Fig. 13)

$$
T \gtrsim T_{\mathrm{ph}}=\frac{\hbar}{k_{\mathrm{B}}} V_{\mathrm{ph}} \Delta k_{\max } \approx 0.6 C T_{\mathrm{p}} Z^{-1 / 3}
$$

the phonon modes with wave numbers $q \sim \Delta k_{\max }$ have nonexponentially small occupation numbers and, hence, the electron-phonon scattering is efficient on the entire Fermi surface. Therefore, even without allowance for the electron-electron collisions, the maximum of the functional (17) defining the electrical conductivity will be reached for smooth trial functions $\Phi_{\mathbf{k}}$. The characteristic temperature $T_{\mathrm{ph}}$ is indicated by the line with short dashes in Fig. 13.

At intermediate temperatures indicated by the gray color in Fig. 13, regions where the quasimomentum diffusion time to the regions of efficient electron-phonon scattering can be larger than the time $\tau$ appear on the Fermi surface. Therefore, the momentum relaxation time in these regions will be determined by the diffusion time to the boundary of the Brillouin zone and not by the Fermi-surfaceaveraged scattering time $\tau$. This can lead to an increase in the electrical conductivity. However, this increase cannot be too large, because the effective diffusion time in the range of temperatures $T_{\text {ee }} \lesssim$ $T \lesssim T_{\text {ph }}$ cannot exceed the time $\tau_{\Delta k}$, which at $\rho \gtrsim$ $10^{11} \mathrm{~g} \mathrm{~cm}^{-3}$ in this temperature range differs from $\tau$ by no more than several times. At $\rho \lesssim 10^{10} \mathrm{~g} \mathrm{~cm}^{-3}$, the difference can be an order of magnitude. Note that this value seems overestimated, because the diffusion time for most of the points on the Fermi surface is much shorter and the actual electrical conductivity difference must be less significant.

In the range $T \ll T_{\mathrm{U}}$ we considered (the temperature $T_{\mathrm{U}}$ is indicated in Fig. 13 by the line with long dashes), the momentum diffusion through electronelectron collisions turns out to be rapid at all densities.

In addition, we disregarded the presence of a magnetic field in the neutron star crust. In the absence of scattering, a magnetic field leads to the rotation of the electron momentum component perpendicular to the magnetic field and, hence, the momentum will regularly approach the lines of intersection between the Fermi sphere and the boundaries of the Brillouin zones. Thus, the deviation of the electron distribution 
function from the equilibrium one will be smoothed out at a sufficient rotation rate:

$$
\begin{gathered}
\tau \gtrsim \tau_{\mathrm{B}}=\omega_{\mathrm{g}}^{-1} \Delta k / k_{\mathrm{F}} \\
\approx 4.3 \times 10^{18} A^{-1 / 3} Z^{-1 / 3} \frac{\rho_{12}}{B_{12}} \mathrm{~s},
\end{gathered}
$$

where $\omega_{\mathrm{g}}=e B /\left(x_{\mathrm{r}} m_{\mathrm{e}} c\right)$ is the electron gyrofrequency and $B_{12}$ is the magnetic field in units of $10^{12} \mathrm{G}$. Note that since the ratio $\Delta k / k_{\mathrm{F}}$ is small, condition (B3) is consistent with the condition for an isotropic electrical conductivity $\tau \omega_{\mathrm{g}} \ll 1$, which is valid for fields $B \lesssim 10^{12} \mathrm{G}$ almost in the entire domain of parameters shown in Fig. 13. In the case of $\tau \omega_{\mathrm{g}} \gtrsim$ 1 , the electrical conductivity becomes anisotropic (Urpin and Yakovlev 1980; Potekhin 1999). Let $T_{\mathrm{B}}$ be the temperature at which $\tau=\tau_{\mathrm{B}}$. The condition $\tau \gtrsim \tau_{\mathrm{B}}$ is then met at $T \lesssim T_{\mathrm{B}}$, because the relaxation time increases with decreasing temperature. The characteristic temperatures $T_{\mathrm{B}}$ for magnetic fields $B=10^{10}, 10^{11}$, and $10^{12} \mathrm{G}$ are indicated in Fig. 13 by the dash-dotted lines. At $B=10^{12} \mathrm{G}$, almost in the entire domain of parameters shown in the figure, the rotation rate of the electron momentum in the magnetic field turns out to be rapid enough for it to cross the boundary of the Brillouin zone at least once in the characteristic electron relaxation time $\tau$. A field of $10^{11} \mathrm{G}$ leads to a fairly rapid smoothing of the distribution function almost in the entire range $T_{\text {ee }} \lesssim T \lesssim T_{\text {ph }}$. The relatively weak (for neutron stars) field of $10^{10} \mathrm{G}$ also reduces considerably the region in which the electron-electron collision rate can limit the momentum relaxation time.

Thus, using a smooth trial function $\Phi_{\mathbf{k}}$ seems justified, at least in the presence of a magnetic field $B \gtrsim 10^{11} \mathrm{G}$. For weak fields in the range $T_{\text {ee }} \lesssim T \lesssim$ $T_{\mathrm{ph}}$, the calculation of the electrical conductivity for matter with equilibrium nuclear composition when using a smooth trial function can underestimate the electrical conductivity, but by no more than an order of magnitude.

We considered the influence of scattering nonuniformity on the Fermi surface with a scale $\sim \Delta k_{\max }$. According to Fig. 12, the density of the lines of intersection between the Fermi sphere and the boundaries of the Brillouin zones (and, hence, the scattering rate) is also nonuniform on a large scale $\sim q_{\mathrm{BZ}}$. However, this nonuniformity is not so strong and should not affect significantly the electrical conductivity. Nevertheless, this conclusion requires an additional verification.

\section{AVERAGING $\left[\left(\mathbf{V}_{\mathbf{k}}-\mathbf{V}_{\mathbf{k}^{\prime}}\right) \cdot \mathbf{u}\right]^{2}$ OVER THE DIRECTIONS OF THE RECIPROCAL LATTICE VECTORS}

The velocity difference $\left(\mathbf{V}_{\mathbf{k}}-\mathbf{V}_{\mathbf{k}^{\prime}}\right)=\mathbf{V}(\mathbf{q})$ is directed along the vector $\mathbf{g}$ (see Section 4 ), while its magnitude is determined by the coordinates of the phonon wave vector $q_{\|}, q_{\perp}$, and $q_{\varphi}$. Therefore, at fixed $q_{\|}, q_{\perp}$, and $q_{\varphi}$, the summation over the vectors $\mathbf{g}_{\mathrm{e}}$ derived from $\mathbf{g}$ by the cubic lattice symmetry transformation (by the reversals and permutations of coordinates) is equivalent to the summation over the vectors derived from $\mathbf{V}(\mathbf{q})$ by the same transformations. Thus,

$$
\begin{aligned}
& \frac{1}{48} \sum_{\mathbf{g}_{\mathrm{e}}}\left[\left(\mathbf{V}_{\mathbf{k}}-\mathbf{V}_{\mathbf{k}^{\prime}}\right) \cdot \mathbf{u}\right]^{2} \\
&= \frac{1}{48}\left\{\left(V_{x} u_{x}+V_{y} u_{y}+V_{z} u_{z}\right)^{2}\right. \\
&+\left(V_{y} u_{x}+V_{x} u_{y}+V_{z} u_{z}\right)^{2} \\
&+\left(-V_{x} u_{x}+V_{z} u_{z}+V_{z} u_{z}\right)^{2} \\
&\left.+\left(-V_{y} u_{x}+V_{x} u_{y}+V_{z} u_{z}\right)^{2}+\ldots\right\} \\
&=\frac{1}{3}\left(\mathbf{V}_{\mathbf{k}}-\mathbf{V}_{\mathbf{k}^{\prime}}\right)^{2} \mathbf{u}^{2}=\frac{1}{3}\left(\mathbf{V}_{\mathbf{k}}-\mathbf{V}_{\mathbf{k}^{\prime}}\right)^{2} .
\end{aligned}
$$

\section{ACKNOWLEDGMENTS}

I thank D.G. Yakovlev for the recommendation of the subject matter for this work and for numerous useful discussions. I am grateful to D.P. Barsukov and M.M. Glazov for valuable consultations. I am also grateful to the anonymous referees for valuable remarks that improved this paper. This work was supported by a grant from the President of Russia for young candidates of science (MK-5857.2010.2), the Russian Foundation for Basic Research (project no. 11-02-00253-a), and the "Leading Scientific Schools of Russia” Program (NSh 3769.2010.2).

\section{REFERENCES}

1. D. A. Baiko, A. D. Kaminker, A. Y. Potekhin, et al., Phys. Rev. Lett. 81, 5556 (1998).

2. D. A. Baiko, A. D. Kaminker, and D. G. Yakovlev, Phys. Rev. E 64, 057402 (2001).

3. D. A. Baiko and D. G. Yakovlev, Astron. Lett. 21, 635 (1995).

4. D. A. Baiko and D. G. Yakovlev, Astron. Lett. 22, 708 (1996).

5. D. A. Baiko, Candidate's Dissertation in Physics and Mathematics (Phys. Tech. Inst. Ioffe, St. Petersburg, 2000).

6. D. A. Baiko, Phys. Rev. E 66, 056405 (2002). 
7. D. P. Barsukov, E. M. Kantor, and A. I. Tsygan, Astron. Rep. 50, 159 (2006).

8. D. P. Barsukov, E. M. Kantor, and A. I. Tsygan, Astron. Rep. 51, 469 (2007).

9. D. P. Barsukov, P. I. Polyakova, and A. I. Tsygan, Astron. Rep. 53, 86 (2009).

10. V. B. Berestetskii, E. M. Lifshits, and L. P. Pitaevskii, Course of Theoretical Physics, Vol. 4: Quantum Electrodynamics (Fizmatlit, Moscow, 2002; Pergamon, Oxford, 1982).

11. V. S. Beskin and E. E. Nokhrina, Astrophys. Space Sci. 308, 589 (2007).

12. P. A. Boldin and S. B. Popov, Mon. Not. R. Astron. Soc. 407, 1090 (2010).

13. A. I. Chugunov and P. Haensel, Mon. Not. R. Astron. Soc. 381, 1143 (2007).

14. A. I. Chugunov and D. G. Yakovlev, Astron. Rep. 49, 724 (2005).

15. E. Flowers and N. Itoh, Astrophys. J. 206, 218 (1976).

16. V. F. Gantmakher and E. A. Levinson, Carrier Scattering in Metals and Semiconductors (Moscow, Nauka 1984; North-Holland, New York, 1987).

17. O. Y. Gnedin, D. G. Yakovlev, and A. Y. Potekhin, Mon. Not. R. Astron. Soc. 324, 725 (2001).

18. M. E. Gusakov, A. D. Kaminker, D. G. Yakovlev, et al., Mon. Not. R. Astron. Soc. 363, 555 (2005)

19. P. Haensel, A. Y. Potekhin, and D. G. Yakovlev, Neutron Stars 1: Equation of State and Structure (Springer-Verlag, New York, 2007).

20. B. Jancovici, Nuovo Cimento 25, 428 (1962).

21. L. D. Landau and I. Ya. Pomeranchuk, Zh. Eksp. Teor. Fiz. 7, 379 (1937).
22. D. R. Lorimer, arXiv:1008.1928 (2010).

23. C. J. Pethick and V. Thorsson, Phys. Rev. D 56, 7548 (1997).

24. J. A. Pons, J. A. Miralles, and U. Geppert, Astron. Astrophys. 496, 207 (2009).

25. S. B. Popov, J. A. Pons, J. A. Miralles, et al., Mon. Not. R. Astron. Soc. 401, 2675 (2010).

26. A. Y. Potekhin, Astron. Astrophys. 351, 787 (1999).

27. A. Y. Potekhin, D. A. Baiko, P. Haensel, et al., Astron. Astrophys. 346, 345 (1999).

28. M. E. Raikh and D. G. Yakovlev, Astrophys. Space Sci. 87, 193 (1982).

29. P. S. Shternin, D. G. Yakovlev, C. O. Heinke, et al., Mon. Not. R. Astron. Soc. Lett. 412, L108 (2011).

30. P. S. Shternin and D. G. Yakovlev, Phys. Rev. D 74, 043004 (2006).

31. P. S. Shternin, J. Phys. A: Math. Theor. 42, 205501 (2008).

32. V. A. Urpin and D. G. Yakovlev, Sov. Astron. 24, 425 (1980).

33. M. Vegelius and A. Melatos, Astrophys. J. 717, 404 (2010).

34. D. G. Yakovlev, W. C. G. Ho, P. S. Shternin, et al., Mon. Not. R. Astron. Soc. 411, 1977 (2011).

35. J. Ziman, Electrons and Photons (Clarendon, Oxford, 1960; Inostr. Liter., Moscow, 1962).

Translated by V. Astakhov 